\title{
ON THE EIGENVARIETY OF HILBERT MODULAR FORMS AT CLASSICAL PARALLEL WEIGHT ONE POINTS WITH DIHEDRAL PROJECTIVE IMAGE
}

\author{
SHAUNAK V. DEO
}

\begin{abstract}
We show that the $p$-adic eigenvariety constructed by AndreattaIovita-Pilloni, parameterizing cuspidal Hilbert modular eigenforms defined over a totally real field $F$, is smooth at certain classical parallel weight one points which are regular at every place of $F$ above $p$ and also determine whether the map to the weight space at those points is étale or not. We prove these results assuming the Leopoldt conjecture for certain quadratic extensions of $F$ in some cases, assuming the $p$-adic Schanuel conjecture in some cases and unconditionally in some cases, using the deformation theory of Galois representations. As a consequence, we also determine whether the cuspidal part of the 1-dimensional parallel weight eigenvariety, constructed by Kisin-Lai, is smooth or not at those points.
\end{abstract}

\section{INTRODUCTION}

In [4], Bellaïche and Dimitrov studied the geometry of the eigencurve of tame level $N$ at classical, regular weight one points and proved that the eigencurve is smooth at all such points. Moreover, they gave a precise criterion for étaleness over the weight space at those points. The main motivation behind their investigation came from questions about specializations of primitive Hida families in weight one like how many families pass through a given weight 1 eigenform, how do those families meet, etc. The aim of this paper is to study the same question for Hilbert modular forms i.e. to study the geometry of the eigenvariety for Hilbert modular forms constructed by Andreatta-Iovita-Pilloni in [1] at classical, regular points of parallel weight 1 .

Before elaborating more on the last paragraph and stating our results, let us fix some notations that we will use throughout the paper. Denote by $G_{L}$ the absolute Galois group of a field $L$. Let $\overline{\mathbb{Q}} \subset \mathbb{C}$ be the field of algebraic numbers. Let $F$ be a totally real field of degree $n$ over $\mathbb{Q}$. Let $i_{1}, \cdots, i_{n}$ denote the distinct complex embeddings of $F$. Fix complex embeddings $m_{1}, \cdots, m_{n}$ of $\overline{\mathbb{Q}}$ such that $m_{j}$ is an extension of $i_{j}$ for all $1 \leq j \leq n$. So, we have complex conjugations $\tau_{1}, \cdots, \tau_{n}$ in $G_{F}$ such that $\tau_{j}$ is the complex conjugation attached to $i_{j}$ i.e. $m_{j}\left(\tau_{j}(x)\right)=\overline{\left(m_{j}(x)\right)}$

2010 Mathematics Subject Classification. 11F80; 11F41(primary).

Key words and phrases. parallel weight one Hilbert modular forms; deformation of Galois representations; eigenvariety. 
for all $1 \leq j \leq n$. Note that $\tau_{1}, \cdots, \tau_{n}$ need not be distinct. Fix a prime number $p$ and an integer $N \geq 4$ such that $p \nmid N$. Let us also fix an algebraic closure $\overline{\mathbb{Q}}_{p}$ of $\mathbb{Q}_{p}$ and an embedding $i_{p}: \overline{\mathbb{Q}} \rightarrow \overline{\mathbb{Q}}_{p}$. Let $\mathfrak{p}_{1}, \cdots, \mathfrak{p}_{r}$ denote the distinct primes of $F$ lying above $p$.

Let $f$ be a cuspidal Hilbert modular eigenform over $F$ of parallel weight one, level $M$ (and tame level $N$ ) and nebentypus $\psi$. So, $\psi$ is a character from $\mathrm{Cl}_{F,+}(M) \rightarrow \mathbb{C}^{*}$, where $\mathrm{Cl}_{F,+}(M)$ is the strict ray class group of $F$ modulo $M$. From the works of Rogawski and Tunnell ([20]) and Wiles ([23]), it follows that there exists a continuous, irreducible representation with finite image: $\rho_{f}: G_{F} \rightarrow G L_{2}(\mathbb{C})$, which is unramified outside $M$ and such that for all primes $\mathfrak{q} \nmid M$, we have $\operatorname{Tr} \rho_{f}\left(\operatorname{Frob}_{\mathfrak{q}}\right)=a(\mathfrak{q}, f)$ and $\operatorname{det} \rho_{f}\left(\operatorname{Frob}_{\mathfrak{q}}\right)=\psi(\mathfrak{q})$, where Frob $\mathfrak{q}_{\mathfrak{q}}$ denotes an arithmetic Frobenius at $\mathfrak{q}$ and $a(\mathfrak{q}, f)$ denotes the eigenvalue of $f$ with respect to the Hecke operator $T(\mathfrak{q})$. Moreover, $\rho_{f}$ is odd in the sense that $\operatorname{det} \rho_{f}\left(\tau_{j}\right)=-1$ for every $1 \leq j \leq n$. For a prime $\mathfrak{p}_{i}$ of $F$ above $p$, let $\alpha_{\mathfrak{p}_{i}}$ and $\beta_{\mathfrak{p}_{i}}$ be the roots of the Hecke polynomial $X^{2}-a\left(\mathfrak{p}_{i}, f\right) X+\psi\left(\mathfrak{p}_{i}\right)$. We say that $f$ is regular at $\mathfrak{p}_{i}$ if $\alpha_{\mathfrak{p}_{i}} \neq \beta_{\mathfrak{p}_{i}}$. We say that $f$ is regular at $p$ if it is regular at all primes of $F$ above $p$ i.e. when $\alpha_{\mathfrak{p}_{i}} \neq \beta_{\mathfrak{p}_{i}}$ for all $1 \leq i \leq r$.

In [1], Andreatta, Iovita and Pilloni constructed the eigenvariety $\mathcal{E}$ for cuspidal Hilbert modular eigenforms of tame level $N$ defined over $F$ with the Hecke operators $U\left(\mathfrak{p}_{i}\right)$ for $i=1, \cdots, r$ and $T(\mathfrak{q})$ for primes $\mathfrak{q} \nmid N p$. The normalization of the operators $U\left(\mathfrak{p}_{i}\right)$ used in [1] to construct $\mathcal{E}$ is different from the classical normalization. The normalization that they use is the same as the one defined by Hida in [13]. Note that these operators coincide with the classical $U\left(\mathfrak{p}_{i}\right)$ 's on parallel weight Hilbert modular forms. See remark 4.7 of [1] and [2, Section 3] for more details. Let $\mathbb{T}$ be $\operatorname{Res}_{\mathcal{O}_{F} / \mathbb{Z}} \mathbb{G}_{m}$ and $M$ be a finite extension of $\mathbb{Q}_{p}$ which splits $F$. There exists a locally finite map $\kappa: \mathcal{E} \rightarrow \mathcal{W}$, where the weight space $\mathcal{W}$ is the rigid analytic space over $M$ associated to the completed group algebra $\mathcal{O}_{M}\left[\left[\mathbb{T}\left(\mathbb{Z}_{p}\right) \times \mathbb{Z}_{p}^{\times}\right]\right]$(see $[1$, Section 2] for more details). In the ordinary case, their construction is same as the construction of Hida families using Katz's p-adic modular forms.

On the other hand, Kisin and Lai ([16]) constructed the parallel weight eigenvariety $\mathcal{C}$ of dimension 1 (which we will call the Kisin-Lai eigencurve) for parallel weight Hilbert modular eigenforms of tame level $N$ defined over $F$ by extending the original construction of the eigencurve for $F=\mathbb{Q}$ given by Coleman and Mazur. The construction of Andreatta, Iovita and Pilloni in the parallel weight case is equivalent to Kisin-Lai's construction (see [1, Section 1]). So, if we denote the weights of $\mathcal{E}$ by $(\nu, w)$ following [1, then we can identify $\mathcal{C}^{\text {cusp }}$, the cuspidal part of the Kisin-Lai eigencurve $\mathcal{C}$, with the closed subspace of $\mathcal{E}$ obtained after making $\nu=0$. 
If $f$ is a classical, cuspidal Hilbert modular form of parallel weight 1 and tame level $N$, then a $p$-stabilization of $f$ with finite slope defines a point on $\mathcal{E}$. By a $p$-stabilization of $f$ with finite slope, we mean an eigenform of tame level $N$ having the same eigenvalues as $f$ away from $p$ and having a non-zero $U_{\mathfrak{p}_{i}}$ eigenvalue for $i=1, \cdots, r$. We denote a $p$-stabilization of $f$ by $f_{\left(\gamma_{i}\right)}$, where $\left(\gamma_{i}\right)$ is an $r$-tuple such that $U_{\mathfrak{p}_{j}} f_{\left(\gamma_{i}\right)}=\gamma_{j} f_{\left(\gamma_{i}\right)}$ for $j=1, \cdots, r$. A $p$-stabilization of $f$ is obtained in the same way as it is obtained in the $F=\mathbb{Q}$ case. So, $\gamma_{i}$ is either $\alpha_{\mathfrak{p}_{i}}$ or $\beta_{\mathfrak{p}_{i}}$ and non-zero for all $1 \leq i \leq r$. Thus, there are at most $2^{r} p$-stabilizations of $f$. As $f$ is of parallel weight 1 , it follows that $U\left(\mathfrak{p}_{i}\right)$-eigenvalue of any $p$-stabilization of $f$ is either a $p$-adic unit or 0 . Thus, if there exists a $p$-stabilization of $f$ with finite slope, then by [23], $\rho_{f}$ is ordinary at $\mathfrak{p}_{i}$ for all $i$. If $f$ is regular at $p$, then $U\left(\mathfrak{p}_{i}\right)$-eigenvalue of any $p$-stabilization is a $p$-adic unit (see [20], [19], [23]). Hence, when $f$ is regular at $p, \rho_{f}$ is ordinary at $\mathfrak{p}_{i}$ for $i=1, \cdots, r$.

Fix a classical, cuspidal Hilbert modular form $f$ over $F$ of parallel weight 1 and tame level $N$ such that it is also regular at $p$. Let $\rho$ be the Galois representation attached to $f$ as above. So, $\left.\rho\right|_{G_{F_{\mathfrak{p}}}}$ is an extension of an unramified character $\psi_{i}^{\prime \prime}$, by a distinct character $\psi_{i}^{\prime}$ for every $1 \leq i \leq r$. For any local Artinian ring $A$ with maximal ideal $\mathfrak{m}_{A}$ and residue field $\overline{\mathbb{Q}}_{p}$, let $\mathcal{D}(A)$ be the set of strict equivalence classes of representations $\rho_{A}: G_{F} \rightarrow G L_{2}(A)$ such that $\rho_{A}\left(\bmod \mathfrak{m}_{A}\right)=\rho$ and which are nearly ordinary at $p$ in the sense that: for every $1 \leq i \leq r$, we have

$$
\left.\rho_{A}\right|_{G_{F_{\mathfrak{p}_{i}}}}=\left(\begin{array}{cc}
\left(\psi_{i}^{\prime}\right)_{A} & * \\
0 & \left(\psi_{i}^{\prime \prime}\right)_{A}
\end{array}\right),
$$

where $\left(\psi_{i}^{\prime \prime}\right)_{A}: G_{F_{\mathfrak{p}_{i}}} \rightarrow A^{\times}$is a character lifting $\psi_{i}^{\prime \prime}$ (nearly ordinary deformation functor). Let $\mathcal{D}_{0}$ be the subfunctor of $\mathcal{D}$ of deformations such that $\left(\psi_{i}^{\prime \prime}\right)_{A}$ is an unramified character of $G_{F_{\mathfrak{p}_{i}}}$ for every $1 \leq i \leq r$ (ordinary deformation functor). Let $\mathcal{D}^{\prime}$ be the subfunctor of $\mathcal{D}_{0}$ of deformations with constant determinant (ordinary deformation functor with constant determinant). We denote the tangent spaces of $\mathcal{D}, \mathcal{D}_{0}, \mathcal{D}^{\prime}$ by $t_{\mathcal{D}}, t_{\mathcal{D}_{0}}$ and $t_{\mathcal{D}^{\prime}}$, respectively.

Let $G$ be the projective image of $\rho$. Denote by ad $\rho$ the adjoint representation of $\rho$ and by $\operatorname{ad}^{0} \rho$ the subspace of $\operatorname{ad} \rho$ of trace zero matrices. Moreover, assume that $G$ is isomorphic to a non-abelian dihedral group. Thus, there exists a unique extension $K$ of $F$ of degree 2 and a finite order character $\chi: G_{K} \rightarrow(\overline{\mathbb{Q}})^{\times}$such that $\rho \simeq \operatorname{Ind}_{G_{K}}^{G_{F}} \chi$. So, we have

$$
\operatorname{ad}^{0} \rho \simeq \epsilon_{K} \oplus \operatorname{Ind}_{G_{K}}^{G_{F}}\left(\chi / \chi^{\sigma}\right)
$$

where $\sigma$ is the non-trivial element of $\operatorname{Gal}(K / F), \epsilon_{K}$ is the quadratic character corresponding to $K$ and $\chi^{\sigma}$ is the character of $G_{K}$ given by $\chi^{\sigma}(g)=\chi\left(\sigma^{-1} g \sigma\right)$. 
Let $n$ be $[F: \mathbb{Q}]$, the degree of $F$ over $\mathbb{Q}$. So, $[K: \mathbb{Q}]=2 n$. Denote by $e_{i}$ the index of ramification and by $f_{i}$ the inertial degree of $\mathfrak{p}_{i}$ for every $1 \leq i \leq r$. Let $S$ be the set of primes of $F$ lying above $p$ which are split in $K$ and $S^{\prime}$ be the set of primes of $F$ which are either inert or ramified in $K$.

We now recall the $p$-adic Schanuel conjecture:

Conjecture 1. (p-adic Schanuel conjecture) Let $\alpha_{1}, \cdots, \alpha_{n}$ be $n$ non-zero algebraic numbers contained in a finite extension $E$ of $\mathbb{Q}_{p}$. Let $\log _{p}: E^{*} \rightarrow E$ be the $p$-adic logarithm normalized so that $\log _{p}(p)=0$. If $\log _{p} \alpha_{1}, \cdots, \log _{p} \alpha_{n}$ are linearly independent over $\mathbb{Q}$, then the extension field $\mathbb{Q}\left(\log _{p} \alpha_{1}, \cdots, \log _{p} \alpha_{n}\right) \subset$ E has transcendence degree $n$ over $\mathbb{Q}$.

(See Conjecture 3.10 of [8])

Theorem 1. Under the assumptions and notations as above, we have:

\begin{tabular}{|c|c|c|c|c|c|c|}
\hline $\begin{array}{l}\text { Condition } \\
\text { on the } \\
\text { degree } n\end{array}$ & $\begin{array}{l}\text { Condition } \\
\text { on } K \text { at } \infty\end{array}$ & $\begin{array}{l}\text { Condition } \\
\text { on } K \text { at } p\end{array}$ & $\begin{array}{l}\text { Transcendence } \\
\text { conjecture as- } \\
\text { sumed }\end{array}$ & $\operatorname{dim} t_{\mathcal{D}}$ & $\operatorname{dim} t_{\mathcal{D}^{\prime}}$ & $\operatorname{dim} t_{\mathcal{D}_{0}}$ \\
\hline- & $\begin{array}{l}K \text { has } 2 n \\
\text { real embed- } \\
\text { dings }\end{array}$ & - & $\begin{array}{l}\text { Leopoldt con- } \\
\text { jecture for } K\end{array}$ & $n+1$ & $\sum_{\mathfrak{p}_{i} \in S} e_{i} f_{i}$ & $\begin{array}{l}\max \\
\left\{\sum_{\mathfrak{p}_{i} \in S} e_{i} f_{i}, 1\right\}\end{array}$ \\
\hline- & $\begin{array}{l}K \quad \text { has } \\
\text { exactly } \\
2(n-1) \\
\text { real embed- } \\
\text { dings }\end{array}$ & - & $\begin{array}{l}\text { Leopoldt con- } \\
\text { jecture for } K\end{array}$ & $n+1$ & $\begin{array}{l}\left(\sum_{\mathfrak{p}_{i} \in S} e_{i} f_{i}\right)- \\
1 \leq \operatorname{dim} t_{\mathcal{D}^{\prime}} \leq \\
(n-1)\end{array}$ & $\begin{array}{l}\max \left\{1, \operatorname{dim} t_{\mathcal{D}^{\prime}}\right\} \\
\leq \operatorname{dim} t_{\mathcal{D}_{0}} \leq \\
\operatorname{dim} t_{\mathcal{D}^{\prime}}+1\end{array}$ \\
\hline- & $\begin{array}{l}K \quad \text { has } \\
\text { exactly } \\
2(n-s) \\
\text { real embed- } \\
\text { dings }\end{array}$ & $\begin{array}{l}\sum_{\mathfrak{p}_{i} \in S} e_{i} f_{i} \\
=n\end{array}$ & $\begin{array}{l}p \text {-adic } \\
\text { Schanuel } \\
\text { conjecture }\end{array}$ & $n+1$ & $n-s$ & $\begin{array}{l}\max \{1, n-s\} \\
\leq \operatorname{dim} t_{\mathcal{D}_{0}} \leq \\
\frac{(n-s)+1}{}\end{array}$ \\
\hline$n=2$ & $\begin{array}{lr}K & \text { has } \\
\text { exactly } 2 \\
\text { real embed- } \\
\text { dings }\end{array}$ & $\begin{array}{l}\sum_{\mathfrak{p}_{i} \in S} e_{i} f_{i} \\
\geq 1\end{array}$ & - & 3 & $\left(\sum_{\mathfrak{p}_{i} \in S} e_{i} f_{i}\right)-$ & 1 \\
\hline
\end{tabular}

TABLE 1.

Remark. (1) From the proof of the theorem above, it will follow that assuming the Leopoldt conjecture for $K$ is not necessary to get the inequalities $\sum_{\mathfrak{p}_{i} \in S} e_{i} f_{i} \leq \operatorname{dim} t_{\mathcal{D}^{\prime}}$ when $K$ is totally real and $\left(\sum_{\mathfrak{p}_{i} \in S} e_{i} f_{i}\right)-1 \leq \operatorname{dim} t_{\mathcal{D}^{\prime}} \leq$ $(n-1)$ when $K$ has exactly $2(n-1)$ real embeddings. Moreover, the proof also implies that the result of Theorem 1 in the first case above, where $K$ 
is totally real, also holds when $G$ is isomorphic to $\mathbb{Z} / 2 \mathbb{Z} \times \mathbb{Z} / 2 \mathbb{Z}$ under the same assumptions and conditions given in the table above.

(2) The fourth case of Theorem 1 above, where $K$ is a real quadratic field and $\sum_{\mathfrak{p}_{i} \in S} e_{i} f_{i} \geq 1$, is a special instance of the second case, where $K$ has exactly $2(n-1)$ real embeddings. Since $\mathcal{O}_{K} \times \otimes_{\mathbb{Z}} \overline{\mathbb{Q}} \simeq 1 \oplus \epsilon_{K}$ as a $G$-representation, it follows from [12, proof of Theorem 1] that the Leopoldt conjecture is indeed true for $K$. So, we don't keep that condition in the fourth case. Moreover, in contrast with the second case, we compute the exact dimension of $t_{\mathcal{D}_{0}}$ in the fourth case without assuming any transcendence conjecture.

(3) Observe that, to compute the dimensions of the tangent spaces in the cases considered in Theorem 1 above, we need to assume the Leopoldt conjecture for $K$ at the very least. But, in [4], Bellaïche and Dimitrov compute the dimensions of these tangent spaces in all the cases when $F=\mathbb{Q}$ without any conditions. Note that, when $F=\mathbb{Q}$ and $f$ is an eigenform such that $\rho_{f} \simeq \operatorname{Ind}_{G_{K}}^{G_{\mathbb{Q}}} \chi$ as above, $K$ is either a real quadratic field or a quadratic imaginary field. Thus, $\operatorname{rank}_{\mathbb{Z}}\left(\mathcal{O}_{K}{ }^{\times}\right)$is either 0 or 1 and hence, the Leopoldt conjecture is true for $K$. Therefore, they don't need to keep that assumption to compute the dimensions in those cases.

Let $f_{\left(\gamma_{i}\right)}$ be a $p$-stabilization of $f$. So, the local ring $\mathcal{T}$ of $\mathcal{E}$ at $f_{\left(\gamma_{i}\right)}$ is a complete noetherian ring with residue field $\overline{\mathbb{Q}}_{p}$ and its Krull dimension is $n+1$. From [1, Theorem 5.1] and the work of Hida ([14]), it follows that there is a nearly ordinary representation $\rho_{\mathcal{T}}: G_{F} \rightarrow G L_{2}(\mathcal{T})$ deforming $\rho$. This induces a map $\mathcal{R} \rightarrow \mathcal{T}$, where $\mathcal{R}$ is the universal deformation ring parameterizing nearly ordinary deformations of $\rho$. Moreover, using results of [14] and arguments of [4] (used in Sec. 5 and 6 of [4]), we see that this map is surjective. We know that the largest quotient of $\mathcal{T}$ on which we get an ordinary deformation of $\rho$ with constant determinant is the algebra $\mathcal{T}^{\prime}$ of the fiber of $\kappa$ at $f_{\left(\gamma_{i}\right)}$ and hence, is of Krull dimension 0. Thus, we get a surjective map $\mathcal{R}^{\prime} \rightarrow \mathcal{T}^{\prime}$, where $\mathcal{R}^{\prime}$ is the universal deformation ring parameterizing ordinary deformations of $\rho$ with constant determinant.

Let $\mathcal{T}_{0}$ be the local ring of $\mathcal{C}^{\text {cusp }}$ at $f_{\left(\gamma_{i}\right)}$. By similar arguments as above, we get an ordinary deformation $\rho_{\mathcal{T}_{0}}: G_{F} \rightarrow G L_{2}\left(\mathcal{T}_{0}\right)$ of $\rho$ and a surjective map $\mathcal{R}_{0} \rightarrow \mathcal{T}_{0}$, where $\mathcal{R}_{0}$ is the universal deformation ring parameterizing ordinary deformations of $\rho$. Since $\mathcal{C}$ has dimension $1, \mathcal{T}_{0}$ is a complete noetherian ring with residue field $\overline{\mathbb{Q}}_{p}$ and Krull dimension 1 . As $\mathcal{C}^{\text {cusp }}$ can be identified with the closed subspace of $\mathcal{E}$ obtained after keeping all the weights same, we get a surjective map $\mathcal{T} \rightarrow \mathcal{T}_{0}$ such 
that the surjective map $\mathcal{T} \rightarrow \mathcal{T}^{\prime}$ factors through it and it is the largest quotient of $\mathcal{T}$ on which we get an ordinary deformation of $\rho$.

Hence, using Theorem 1 along with the discussion in the preceding paragraphs, we obtain the following results regarding the geometry of $\mathcal{E}$ and $\mathcal{C}^{\text {cusp }}$ at $f_{\left(\gamma_{i}\right)}$ satisfying the conditions of Theorem 1 ;

Theorem 2. Suppose $K$ has at least $2(n-1)$ real embeddings and the Leopoldt conjecture is true for $K$. Then, the eigenvariety $\mathcal{E}$ is smooth at $f_{\left(\gamma_{i}\right)}$. Moreover:

(1) If $K$ is totally real, then the weight map $\kappa$ is étale at $f_{\left(\gamma_{i}\right)}$ if and only if $\sum_{\mathfrak{p}_{i} \in S} e_{i} f_{i}=0$ (i.e. no prime of $F$ lying above $p$ is split in $K$ ) and the parallel weight cuspidal eigenvariety $\mathcal{C}^{\text {cusp }}$ is smooth at $f_{\left(\gamma_{i}\right)}$ if and only if $\sum_{\mathfrak{p}_{i} \in S} e_{i} f_{i} \leq 1$

(2) If $K$ has exactly $2(n-1)$ real embeddings, then the weight map $\kappa$ is not étale at $f_{\left(\gamma_{i}\right)}$ if $\sum_{\mathfrak{p}_{i} \in S} e_{i} f_{i} \geq 2$ and the parallel weight cuspidal eigenvariety $\mathcal{C}^{\text {cusp }}$ is not smooth at $f_{\left(\gamma_{i}\right)}$ if $\sum_{\mathfrak{p}_{i} \in S} e_{i} f_{i} \geq 3$.

Theorem 3. Suppose all the primes of $F$ lying above $p$ are split in $K$ and the p-adic Schanuel conjecture is true. Then: the eigenvariety $\mathcal{E}$ is smooth at $f_{\left(\gamma_{i}\right)}$. The weight map $\kappa$ is étale at $f_{\left(\gamma_{i}\right)}$ if and only if $K$ is a CM field. The parallel weight cuspidal eigenvariety $\mathcal{C}^{\text {cusp }}$ is not smooth at $f_{\left(\gamma_{i}\right)}$ if $K$ has at least 4 real embeddings.

Theorem 4. Suppose $F$ is a real quadratic field. If $K$ is not a totally real or $C M$ field and $\sum_{\mathfrak{p}_{i} \in S} e_{i} f_{i} \geq 1$, then the eigenvarieties $\mathcal{E}$ and $\mathcal{C}^{\text {cusp }}$ are smooth at $f_{\left(\gamma_{i}\right)}$. The weight map $\kappa$ is étale at $f_{\left(\gamma_{i}\right)}$ if and only if $\sum_{\mathfrak{p}_{i} \in S} e_{i} f_{i}=1$.

We recently learned that A. Betina has announced results similar to part 1 of Theorem 2 and Theorem 3 in [5], using methods which are different from the ones presented here.

The non-smoothness of $\mathcal{C}^{\text {cusp }}$ at $f_{\left(\gamma_{i}\right)}$ implies the non-smoothness of $\mathcal{C}$ at $f_{\left(\gamma_{i}\right)}$. Note that, we can use the arguments of [4, Section 7] in the cases considered above to prove that local rings of the full eigenvarieties $\mathcal{E}^{\text {full }}$ and $\left(\mathcal{C}^{\text {cusp }}\right)^{\text {full }}$ at $f_{\left(\gamma_{i}\right)}$ are isomorphic to $\mathcal{T}$ and $\mathcal{T}_{0}$, respectively. However we need the results of [9], 22], 20], [19], [15] to use the arguments of [4]. This allows us to conclude that there is a unique, up to Galois conjugacy, nearly-ordinary Hida family passing through $f_{\left(\gamma_{i}\right)}$ satisfying the conditions of Theorem 1 and we may also get examples of $f_{\left(\gamma_{i}\right)}$ with only one Galois orbit of ordinary Hida families passing through it (smooth points of $\mathcal{C}$ ). Moreover, the existence of non-smooth points of $\mathcal{C}$ indicates the possibility of getting examples of $f_{\left(\gamma_{i}\right)}$ with at least two non-Galois conjugate ordinary Hida families passing through it. 
In [11], Darmon, Lauder and Rotger used the non-étaleness of the weight map of the $p$-adic Coleman-Mazur eigencurve at classical, regular weight one points with real multiplication by a quadratic real field $F$ in which $p$ is split (which is proved by Cho-Vatsal ([10]) and Bellaïche-Dimitrov ([4])) to find overconvergent $p$-adic modular forms of weight 1 whose Fourier coefficients can be expressed as $p$-adic logarithms of algebraic numbers lying in ring class fields of $F$. The non-étaleness results that we obtain here could be used to get results in a similar direction for number fields of higher degree over $\mathbb{Q}$ which are not necessarily totally real (in fact a similar result for totally real fields has been announced by Betina in [5]).

Taking an inspiration from Theorem 1, we make the following conjecture:

Conjecture 2. Keeping the assumptions and notations established just before Theorem 1, suppose $K$ has exactly $2(n-s)$ real embeddings. Then, $\operatorname{dim} t_{\mathcal{D}}=n+1$ and $\operatorname{dim} t_{\mathcal{D}^{\prime}}=\max \left\{\left(\sum_{\mathfrak{p}_{i} \in S} e_{i} f_{i}\right)-s, 0\right\}$.

As a consequence of the conjecture above, we get the following result:

Let $f$ be a Hilbert modular form over $F$ satisfying the properties of Conjecture 2 and $f_{\left(\gamma_{i}\right)}$ be a $p$-stabilization of $f$. Then, the eigenvariety $\mathcal{E}$ is smooth at $f_{\left(\gamma_{i}\right)}$. The weight map $\kappa$ is étale at $f_{\left(\gamma_{i}\right)}$ if and only if $\left(\sum_{\mathfrak{p}_{i} \in S} e_{i} f_{i}\right)-s \leq 0$.

Let us give an outline of the techniques used to prove Theorem 1 . We identify $t_{\mathcal{D}^{\prime}}$ as a certain subspace of $H^{1}\left(F, \operatorname{ad}^{0} \rho\right)$ and $t_{\mathcal{D}_{0}}, t_{\mathcal{D}}$ as certain subspaces of $H^{1}(F, \operatorname{ad} \rho)$. As $\rho$ has finite image, following [4], we apply the inflation-restriction sequences to see the tangent spaces as subspaces of $\left(\operatorname{Hom}\left(G_{H}, \overline{\mathbb{Q}}_{p}\right) \otimes \operatorname{ad} \rho\right)^{G}$, where $H$ is the finite Galois extension of $F$ cut out by ad $\rho$. We use the fact that $G$ is a non-abelian dihedral group to get an explicit description of the possible elements of these spaces in the cases mentioned in Theorem 1. After getting the explicit description, we employ the technique of using algebraic subspace of $\operatorname{Hom}\left(G_{H}, \overline{\mathbb{Q}}_{p}\right)$ of [4] to get an upper bound on the dimension of tangent spaces and use the structure of $\mathcal{O}_{H}^{\times} \otimes_{\mathbb{Z}} \overline{\mathbb{Q}}$ as a $G$-representation to get a lower bound on the dimension of tangent spaces whenever possible. In some cases, this already gives us the dimension of some tangent spaces, for instance, the dimension of $t_{\mathcal{D}^{\prime}}$ in the second case of Theorem 1 when all primes of $F$ lying above $p$ are split in $K$. We then compute the dimensions of remaining tangent spaces with the help of explicit description of the elements of tangent spaces, techniques of [4] and some transcendence results.

The transcendence results that we use to compute the dimension are the BakerBrumer theorem, the Leopoldt conjecture and the $p$-adic Schanuel conjecture. They can be ordered from weakest to strongest, with the Baker-Brumer theorem being the 
weakest and the $p$-adic Schanuel conjecture being the strongest. In fact, the $p$-adic Schanuel conjecture implies the Leopoldt conjecture (see Theorem 6.4 of [17]). We use only one of the Baker-Brumer theorem, the Leopoldt conjecture and the $p$-adic Schanuel conjecture depending on the case in hand. More specifically, we try to give the proofs using the weakest possible results of the above. It turns out that, in some cases the weakest of the transcendence results (the Baker-Brumer theorem) is sufficient, but in some cases, we have to assume the strongest of the transcendence results (the $p$-adic Schanuel conjecture) to conclude our results.

Acknowledgments. This work was done as a part of my doctoral thesis at Brandeis University. I would like to thank my Ph.D. advisor Joël Bellaïche for suggesting this problem to me and for all the help and encouragement he provided during the completion of this paper. He also provided some crucial inputs to make the exposition clearer. This paper would not have been possible without his help. I would like to thank Vincent Pilloni and Adrian Iovita for useful communications about their paper ([1]). I would like to thank Chandrashekhar Khare for helpful conversations regarding this paper and for directing me to [17]. I would also like to thank Aditya Karnataki and John Bergdall for many mathematical discussions. I would like to thank the referee as well for a careful reading and helpful suggestions.

\section{TANGENT SPACES OF NEARLY ORDINARY AND ORDINARY DEFORMATION PROBLEMS}

We keep the notations from the previous section. Even though all our main theorems are for finite image representations (coming from classical, cuspidal Hilbert modular eigenforms of parallel weight 1 which are regular at $p$ ) with dihedral projective image, the results that we state here and in the next sections ( $\S 3$ and $\S 4$ ) will also hold for a general finite image representation (coming from classical, cuspidal Hilbert modular eigenforms of parallel weight 1 which are regular at $p$ ) unless specified otherwise. So, in what follows, we keep all the assumptions on $\rho$ from the previous section except the assumption of projective dihedral image. We will be following [4] closely in this section and in the next two sections.

2.1. Relations with the cohomology groups. As $\rho$ has finite image, it is equivalent to a representation whose image is in $G L_{2}(\overline{\mathbb{Q}})$. Using the embedding $i_{p}$, we can see $\rho$ as a representation of $G_{F}$ on a 2 -dimensional $\overline{\mathbb{Q}}_{p}$-vector space $V$. Recall that, for a prime $\mathfrak{p}_{i}$ of $F$ lying above $p,\left.\rho\right|_{G_{\mathfrak{p}_{i}}}$ is an extension of an unramified character $\psi_{i}^{\prime \prime}$ by a distinct character $\psi_{i}^{\prime}$. Since $\rho$ has finite image, we can fix a basis $\left(e_{1, i}, e_{2, i}\right)$ of $V$ for which $\rho\left(G_{F}\right) \subset G L_{2}(\overline{\mathbb{Q}})$ and such that $\left.\rho\right|_{G_{\mathfrak{p}_{i}}}$ acts by the character 
$\psi_{i}^{\prime}$ on $\overline{\mathbb{Q}}_{p} e_{1, i}$ and by $\psi_{i}^{\prime \prime}$ on $\overline{\mathbb{Q}}_{p} e_{2, i}$. This basis is well-defined up to a scaling in the sense that if $\left(f_{1, i}, f_{2, i}\right)$ is another such basis, then there exist $x_{i}, y_{i} \in(\overline{\mathbb{Q}})^{\times}$such that $f_{1, i}=x_{i} e_{1, i}$ and $f_{2, i}=y_{i} e_{2, i}$. Note that this basis may be different for different primes of $F$ lying above $p$. Thus, we have a set of $r$ possibly distinct bases, one for each prime of $F$ lying above $p$.

Choosing each basis $\left(e_{1, i}, e_{2, i}\right)$ of $V$ as above, we can identify $\operatorname{End}_{\overline{\mathbb{Q}}_{p}}(V)$ with $M_{2}\left(\overline{\mathbb{Q}}_{p}\right)$. Hence, we get four continuous maps $A_{i}, B_{i}, C_{i}, D_{i}: \operatorname{End}_{\overline{\mathbb{Q}}_{p}}(V) \rightarrow \overline{\mathbb{Q}}_{p}$ given by the upper-left, upper-right, lower-left and lower-right entries of matrices, respectively. By definition of the basis $\left(e_{1, i}, e_{2, i}\right)$, these maps are morphisms of $G_{F_{\mathfrak{p}_{i}}}$ as follows:

$$
\begin{aligned}
& A_{i}:\left.(\operatorname{ad} \rho)\right|_{G_{F_{\mathfrak{p}_{i}}}} \rightarrow \overline{\mathbb{Q}}_{p}, B_{i}:\left.(\operatorname{ad} \rho)\right|_{G_{F_{\mathfrak{p}_{i}}}} \rightarrow \overline{\mathbb{Q}}_{p}\left(\psi_{i}^{\prime} / \psi_{i}^{\prime \prime}\right) \\
& C_{i}:\left.(\operatorname{ad} \rho)\right|_{G_{F_{\mathfrak{p}_{i}}}} \rightarrow \overline{\mathbb{Q}}_{p}\left(\psi_{i}^{\prime \prime} / \psi_{i}^{\prime}\right), D_{i}:\left.(\operatorname{ad} \rho)\right|_{G_{F_{\mathfrak{p}_{i}}}} \rightarrow \overline{\mathbb{Q}}_{p}
\end{aligned}
$$

where $\overline{\mathbb{Q}}_{p}\left(\psi_{i}^{\prime \prime} / \psi_{i}^{\prime}\right)$ denotes the 1-dimensional representation of $G_{F_{\mathfrak{p}_{i}}}$ on $\overline{\mathbb{Q}}_{p}$ coming from the character $\psi_{i}^{\prime \prime} / \psi_{i}^{\prime}$ and $\overline{\mathbb{Q}}_{p}\left(\psi_{i}^{\prime} / \psi_{i}^{\prime \prime}\right)$ is defined similarly.

Recall that we have chosen an embedding $G_{F_{\mathfrak{p}_{i}}} \hookrightarrow G_{F}$, which gives us the restriction morphism $H^{1}(F, \operatorname{ad} \rho) \rightarrow H^{1}\left(F_{\mathfrak{p}_{i}}\right.$, ad $\left.\rho\right)$. Composing this restriction morphism with the map $H^{1}\left(F_{\mathfrak{p}_{i}}\right.$, ad $\left.\rho\right) \rightarrow H^{1}\left(F_{\mathfrak{p}_{i}}, \overline{\mathbb{Q}}_{p}\left(\psi_{i}^{\prime \prime} / \psi_{i}^{\prime}\right)\right)$ induced by $C_{i}$, we get a homomorphism

$$
C_{i, *}: H^{1}(F, \operatorname{ad} \rho) \rightarrow H^{1}\left(F_{\mathfrak{p}_{i}}, \overline{\mathbb{Q}}_{p}\left(\psi_{i}^{\prime \prime} / \psi_{i}^{\prime}\right)\right)
$$

Denote by $I_{\mathfrak{p}_{i}}$ the inertia group at $\mathfrak{p}_{i}$. By composing the restriction morphism $H^{1}(F, \operatorname{ad} \rho) \rightarrow H^{1}\left(I_{\mathfrak{p}_{i}}, \operatorname{ad} \rho\right)$ with the map $H^{1}\left(I_{\mathfrak{p}_{i}}, \operatorname{ad} \rho\right) \rightarrow H^{1}\left(I_{\mathfrak{p}_{i}}, \overline{\mathbb{Q}}_{p}\right)$ induced by $D_{i}$, we get a homomorphism

$$
D_{i, *}: H^{1}(F, \operatorname{ad} \rho) \rightarrow H^{1}\left(I_{\mathfrak{p}_{i}}, \overline{\mathbb{Q}}_{p}\right)
$$

As in the introduction, let $\mathcal{D}, \mathcal{D}_{0}, \mathcal{D}^{\prime}$ be the nearly-ordinary deformation functor, ordinary and ordinary deformation functor with constant determinant of $\rho$, respectively and their tangent spaces be $t_{\mathcal{D}}, t_{\mathcal{D}_{0}}$ and $t_{\mathcal{D}^{\prime}}$, respectively.

Lemma 1. We have:

(1) $t_{\mathcal{D}^{\prime}}=\operatorname{ker}\left(H^{1}\left(F, a d^{0} \rho\right) \stackrel{\left(\left(C_{i, *}\right),\left(D_{i, *}\right)\right)}{\longrightarrow} \oplus_{i=1}^{r} H^{1}\left(F_{\mathfrak{p}_{i}}, \overline{\mathbb{Q}}_{p}\left(\psi_{i}^{\prime \prime} / \psi_{i}^{\prime}\right)\right) \bigoplus \bigoplus_{i=1}^{r} H^{1}\left(I_{\mathfrak{p}_{i}}, \overline{\mathbb{Q}}_{p}\right)\right)$.

(2) $t_{\mathcal{D}_{0}}=\operatorname{ker}\left(H^{1}(F, a d \rho) \stackrel{\left(\left(C_{i, *}\right),\left(D_{i, *}\right)\right)}{\longrightarrow} \oplus_{i=1}^{r} H^{1}\left(F_{\mathfrak{p}_{i}}, \overline{\mathbb{Q}}_{p}\left(\psi_{i}^{\prime \prime} / \psi_{i}^{\prime}\right)\right) \bigoplus \bigoplus_{i=1}^{r} H^{1}\left(I_{\mathfrak{p}_{i}}, \overline{\mathbb{Q}}_{p}\right)\right)$.

(3) $t_{\mathcal{D}}=\operatorname{ker}\left(H^{1}(F, a d \rho) \stackrel{\left(C_{i, *}\right)}{\longrightarrow} \oplus_{i=1}^{r} H^{1}\left(F_{\mathfrak{p}_{i}}, \overline{\mathbb{Q}}_{p}\left(\psi_{i}^{\prime \prime} / \psi_{i}^{\prime}\right)\right)\right)$.

Proof. Same as the proof of Lemma 2.3 of [4]. We just need to repeat their argument for every prime $\mathfrak{p}_{i}$ of $F$ lying above $p$. 
2.2. Application of inflation-restriction. Let $H$ be a finite Galois extension of $F$ with $G=\operatorname{Gal}(H / F)$. The choice of decomposition group at every prime $\mathfrak{p}_{i}$ of $F$ above $p$ singles out a prime $w_{i}$ among the primes of $H$ lying above $\mathfrak{p}_{i}$ along with embeddings $G_{H_{w_{i}}} \subset G_{H}$ and $G_{H_{w_{i}}} \subset G_{F_{\mathfrak{p}_{i}}}$. Let $W$ be a $G_{F^{-}}$-representation on a finite dimensional $\overline{\mathbb{Q}}_{p}$-vector space.

Lemma 2. For every $i$ between 1 and $r$, let $W_{1, i}$ be a quotient of $W$ as a $G_{F_{\mathfrak{p}_{i}}}$ representation and $W_{2, i}$ be a quotient of $W$ as a $I_{\mathfrak{p}_{i}}$-representation. Then the restriction morphism yields the following isomorphisms:

(1) $\operatorname{ker}\left(H^{1}(F, W) \rightarrow \oplus_{i=1}^{r} H^{1}\left(F_{\mathfrak{p}_{i}}, W_{1, i}\right) \bigoplus \oplus_{i=1}^{r} H^{1}\left(I_{\mathfrak{p}_{i}}, W_{2, i}\right)\right) \stackrel{\simeq}{\rightarrow} \operatorname{ker}\left(H^{1}(H, W)^{G} \rightarrow\right.$ $\left.\oplus_{i=1}^{r} H^{1}\left(H_{w_{i}}, W_{1, i}\right) \bigoplus \oplus_{i=1}^{r} H^{1}\left(I_{w_{i}}, W_{2, i}\right)\right)$.

(2) $\operatorname{ker}\left(H^{1}(F, W) \rightarrow \oplus_{i=1}^{r} H^{1}\left(F_{\mathfrak{p}_{i}}, W_{1, i}\right)\right) \stackrel{\simeq}{\rightarrow} \operatorname{ker}\left(H^{1}(H, W)^{G} \rightarrow \oplus_{i=1}^{r} H^{1}\left(H_{w_{i}}, W_{1, i}\right)\right)$.

Proof. Same as the proof of Lemma 2.4 of [4] which works because $H$ is a finite extension of $F$.

Now we take $H$ to be the subfield of $\overline{\mathbb{Q}}$ fixed by $\operatorname{ker}(\operatorname{ad} \rho)$. Then $G=\operatorname{Gal}(H / F)$ is naturally identified with the projective image of $\rho$ which we will call $\operatorname{Proj}(\rho)$. We will now fix this notation for the rest of the paper. After choosing a suitable basis $\left(v_{1}, v_{2}\right)$, we can view $\operatorname{Proj}(\rho)(g)$ as an element of $P G L_{2}(\overline{\mathbb{Q}})$ for every $g \in G$. For a matrix $Y \in M_{2}\left(\overline{\mathbb{Q}}_{p}\right)$, by abuse of notation, we shall denote by $\rho(g) Y \rho(g)^{-1}$ the image of $Y$ by the adjoint action of $\operatorname{Proj}(\rho)(\mathrm{g})$.

As $\rho(H)=1$, it follows that $H^{1}(H, \operatorname{ad} \rho)=H^{1}\left(H, \overline{\mathbb{Q}}_{p}\right) \otimes_{\overline{\mathbb{Q}}_{p}}$ ad $\rho$. We can write an element of $H^{1}\left(H, \overline{\mathbb{Q}}_{p}\right) \otimes_{\overline{\mathbb{Q}}_{p}}$ ad $\rho$ as $\left(\begin{array}{ll}a & b \\ c & d\end{array}\right)$ where $a, b, c, d \in H^{1}\left(H, \overline{\mathbb{Q}}_{p}\right)$. The natural left action of $G$ on $H^{1}\left(H, \overline{\mathbb{Q}}_{p}\right) \otimes_{\overline{\mathbb{Q}}_{p}}$ ad $\rho$ is given by:

$$
g \cdot\left(\begin{array}{ll}
a & b \\
c & d
\end{array}\right)=\rho(g)\left(\begin{array}{cc}
g \cdot a & g \cdot b \\
g \cdot c & g \cdot d
\end{array}\right) \rho(g)^{-1}
$$

Hence, as $H^{1}(F, \operatorname{ad} \rho) \simeq\left(H^{1}\left(H, \overline{\mathbb{Q}}_{p}\right) \otimes_{\overline{\mathbb{Q}}_{p}} \operatorname{ad} \rho\right)^{G}$, an element of $H^{1}(F, \operatorname{ad} \rho)$ is just a matrix $\left(\begin{array}{ll}a & b \\ c & d\end{array}\right)$ as above which is $G$-invariant. Note that, if we change the basis $\left(v_{1}, v_{2}\right)$, then it may change the image of $\operatorname{Proj}(\rho)$ in $P G L_{2}\left(\overline{\mathbb{Q}}_{p}\right)$. Thus, this may also change the matrix presentation of elements of $H^{1}(F, \operatorname{ad} \rho)$ given above. To be precise, if we choose a different basis $\left(v_{1}^{\prime}, v_{2}^{\prime}\right)$ and if $P$ is the change of basis matrix, then an element of $H^{1}(F, \operatorname{ad} \rho)$ represented by the matrix $\left(\begin{array}{ll}a & b \\ c & d\end{array}\right)$ under the original basis $\left(v_{1}, v_{2}\right)$ as above will now be represented by the matrix $P\left(\begin{array}{ll}a & b \\ c & d\end{array}\right) P^{-1}$. 
If an element of $H^{1}(F, \operatorname{ad} \rho)$ is represented by $\left(\begin{array}{ll}a & b \\ c & d\end{array}\right)$ under the chosen basis $\left(v_{1}, v_{2}\right)$, then denote its matrix presentation under the basis $\left(e_{1, i}, e_{2, i}\right)$ by $\left(\begin{array}{ll}a_{(i)} & b_{(i)} \\ c_{(i)} & d_{(i)}\end{array}\right)$ for $i=1, \cdots, r$. From the discussion above, we see that $a_{(i)}, b_{(i)}, c_{(i)}$ and $d_{(i)}$ are just $\overline{\mathbb{Q}}_{p}$-linear combinations of $a, b, c$ and $d$ for every $i$. If the image of the change of basis matrix corresponding to $\left(e_{1, i}, e_{2, i}\right)$ in $P G L_{2}\left(\overline{\mathbb{Q}}_{p}\right)$ lies in $P G L_{2}(\overline{\mathbb{Q}})$, then $a_{(i)}, b_{(i)}, c_{(i)}$ and $d_{(i)}$ are in fact $\overline{\mathbb{Q}}$-linear combinations of $a, b, c$ and $d$. Combining all the discussion above along with the previous two lemmas, we get the following lemma:

Lemma 3. Denote the morphism sending $\left(\begin{array}{ll}a & b \\ c & d\end{array}\right) \in H^{1}(H$, ad $\rho)$ to the restriction of $c_{(i)}$ to $G_{H_{w_{i}}}$ by $\phi_{i}$ and the morphism sending the same element to the restriction of $d_{(i)}$ to $I_{w_{i}}$ by $\phi_{i}^{\prime}$. These morphisms yield the following isomorphisms:

$$
\begin{gathered}
t_{\mathcal{D}}=\operatorname{ker}\left(\left(H^{1}\left(H, \overline{\mathbb{Q}}_{p}\right) \otimes_{\overline{\mathbb{Q}}_{p}} a d \rho\right)^{G} \stackrel{\left(\phi_{i}\right)}{\longrightarrow} \oplus_{i=1}^{r} H^{1}\left(H_{w_{i}}, \overline{\mathbb{Q}}_{p}\right)\right) \\
t_{\mathcal{D}_{0}}=\operatorname{ker}\left(\left(H^{1}\left(H, \overline{\mathbb{Q}}_{p}\right) \otimes_{\overline{\mathbb{Q}}_{p}} a d \rho\right)^{G} \stackrel{\left(\left(\phi_{i}\right),\left(\phi_{i}^{\prime}\right)\right)}{\longrightarrow} \oplus_{i=1}^{r} H^{1}\left(H_{w_{i}}, \overline{\mathbb{Q}}_{p}\right) \oplus \oplus_{i=1}^{r} H^{1}\left(I_{w_{i}}, \overline{\mathbb{Q}}_{p}\right)\right) \\
t_{\mathcal{D}^{\prime}}=\operatorname{ker}\left(\left(H^{1}\left(H, \overline{\mathbb{Q}}_{p}\right) \otimes_{\overline{\mathbb{Q}}_{p}} a d^{0} \rho\right)^{G} \stackrel{\left(\left(\phi_{i}\right),\left(\phi_{i}^{\prime}\right)\right)}{\longrightarrow} \oplus_{i=1}^{r} H^{1}\left(H_{w_{i}}, \overline{\mathbb{Q}}_{p}\right) \oplus \oplus_{i=1}^{r} H^{1}\left(I_{w_{i}}, \overline{\mathbb{Q}}_{p}\right)\right)
\end{gathered}
$$

\section{Structure of $H^{1}\left(H, \overline{\mathbb{Q}}_{p}\right)$ As a $G$-Representation}

We shall denote by $\mathcal{O}_{H}$ the ring of integers of $H$ and by $\hat{G}$ the set of equivalence classes of left irreducible representations of $G=\operatorname{Gal}(H / F)$ over $\overline{\mathbb{Q}}$ or over $\overline{\mathbb{Q}}_{p}$ (the two sets can be identified using the embedding $i_{p}$ ). We shall denote the trivial representation of $G$ by 1 .

3.1. Local units. It is known that $\mathcal{O} \otimes_{\mathbb{Z}} \mathbb{Z}_{p} \simeq \prod_{w \mid p} \mathcal{O}_{H_{w}}$ where $w$ runs over all places of $H$ above $p$ and $\mathcal{O}_{H_{w}}$ is the ring of integers of the completion $H_{w}$.

By local class field theory, the image of the restriction homomorphism $\operatorname{Hom}\left(G_{H_{w}}, \overline{\mathbb{Q}}_{p}\right) \rightarrow$ $\operatorname{Hom}\left(I_{w}, \overline{\mathbb{Q}}_{p}\right)$ is isomorphic to $\operatorname{Hom}\left(\mathcal{O}_{H_{w}}^{\times}, \overline{\mathbb{Q}}_{p}\right)$. Let $\log _{p}: \overline{\mathbb{Q}}_{p}^{\times} \rightarrow \overline{\mathbb{Q}}_{p}$ be the standard $p$-adic logarithm sending $p$ to 0 . A continuous homomorphism $\mathcal{O}_{H_{w}}^{\times} \rightarrow \overline{\mathbb{Q}}_{p}$ is of the form

$$
u \mapsto \sum_{s_{w} \in J_{w}} h_{s_{w}} g_{w}\left(\log _{p}(u)\right)=\sum_{s_{w} \in J_{w}} h_{s_{w}} \log _{p}\left(g_{w}(u)\right)
$$

for some $h_{s_{w}} \in \overline{\mathbb{Q}}_{p}$, where $J_{w}$ is the set of all embeddings of $H_{w}$ in $\overline{\mathbb{Q}}_{p}$. 
Let $S$ be the set of all embeddings of $H$ in $\overline{\mathbb{Q}}$. The commutative diagram

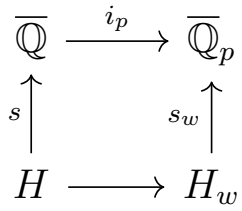

along with the embedding $\overline{\mathbb{Q}} \subset \mathbb{C}$ defines a partition

$$
S=\bigsqcup_{w \mid p} J_{w}
$$

Let $i_{1}^{\prime}, \cdots, i_{n}^{\prime}$ be embeddings of $H$ in $\overline{\mathbb{Q}}$ such that they lift the embeddings $i_{1}, \cdots, i_{n}$ of $F$ in $\overline{\mathbb{Q}}$ and in the partition of $S$ given above, they lie in the set $\bigsqcup_{w_{i}} J_{w_{i}}$ (i.e. when we compose any of these embeddings with $i_{p}$, the place of $H$ above $p$ that it chooses in the diagram above is one of the $w_{i}$ 's). The existence of such embeddings is clear. Note that, we also have $S=\bigsqcup_{j=1}^{n} i_{1}^{\prime} \circ G$. Hence, the $\overline{\mathbb{Q}}_{p}$ vector space $\operatorname{Hom}\left(\left(\mathcal{O}_{H} \otimes_{\mathbb{Z}} \mathbb{Z}_{p}\right)^{\times}, \overline{\mathbb{Q}}_{p}\right)$ has a canonical basis given by $\left(\log _{p}\left(i_{p} \circ i_{j}^{\prime} \circ g \otimes 1\right)\right)_{g \in G, 1 \leq j \leq n}$. As $g^{\prime} \in G$ acts on the left on this basis sending $\log _{p}\left(i_{p} \circ i_{j}^{\prime} \circ g \otimes 1\right)$ to $\log _{p}\left(i_{p} \circ i_{j}^{\prime} \circ g^{\prime} g \otimes 1\right)$, we get a canonical isomorphism of left $G$-representations:

$$
\begin{gathered}
\oplus_{j=1}^{n} \overline{\mathbb{Q}}_{p}[G] \rightarrow \operatorname{Hom}\left(\left(\mathcal{O}_{H} \otimes \mathbb{Z}_{p}\right)^{\times}, \overline{\mathbb{Q}}_{p}\right) \\
\sum_{j=1}^{n} \sum_{g \in G} h_{g, j} g \mapsto\left(u \otimes v \mapsto \sum_{j=1}^{n} \sum_{g \in G} h_{g, j} \log _{p}\left(i_{p}\left(i_{j}\left(g^{-1}(u)\right)\right) v\right)\right)
\end{gathered}
$$

3.2. Global units and $\operatorname{Hom}\left(G_{H}, \overline{\mathbb{Q}}_{p}\right)$. By global class field theory, we have the following exact sequence of left $\overline{\mathbb{Q}}_{p}[G]$-modules:

$$
0 \rightarrow \operatorname{Hom}\left(G_{H}, \overline{\mathbb{Q}}_{p}\right) \rightarrow \operatorname{Hom}\left(\left(\mathcal{O}_{H} \otimes \mathbb{Z}_{p}\right)^{\times}, \overline{\mathbb{Q}}_{p}\right) \rightarrow \operatorname{Hom}\left(\mathcal{O}_{H}^{\times}, \overline{\mathbb{Q}}_{p}\right)
$$

where the first map is dual to the Artin reciprocity map, and the second is the restriction with respect to the inclusion $\mathcal{O}_{H}^{\times} \rightarrow\left(\mathcal{O}_{H} \otimes \mathbb{Z}_{p}\right)^{\times}, u \mapsto u \otimes 1$. The surjectivity of the last map above is equivalent to the Leopoldt conjecture for $H$.

By Minkowski's proof of Dirichlet's unit theorem, we get the following isomorphism of left $G$-representations:

$$
\operatorname{Hom}\left(\mathcal{O}_{H}^{\times}, \overline{\mathbb{Q}}_{p}\right) \simeq \oplus_{j=1}^{n}\left(\operatorname{Ind}_{\left\{1, \tau_{j}\right\}}^{G} 1\right) \backslash 1
$$

For $\pi \in \hat{G}$, let $\pi^{\{+, j\}}$ be the subspace of $\pi$ on which $\tau_{j}$ acts by 1 and $\pi^{\{-, j\}}$ be the subspace of $\pi$ on which $\tau_{j}$ acts by -1 . So, using this notation, we have $\operatorname{Hom}\left(\mathcal{O}_{H}^{\times}, \overline{\mathbb{Q}}_{p}\right) \simeq \oplus_{\pi \in \hat{G}, \pi \neq 1} \pi^{\left(\sum_{j=1}^{n} \operatorname{dim} \pi^{\{+, j\}}\right)} \oplus 1^{(n-1)}$ as a left $G$-representation. Hence, as a left $G$-representation, we have $\operatorname{Hom}\left(G_{H}, \overline{\mathbb{Q}}_{p}\right) \simeq \oplus_{\pi \in \hat{G}} \pi^{m_{\pi}}$, with $m_{1} \geq 1$ and $\sum_{j=1}^{n} \operatorname{dim} \pi^{\{-, j\}} \leq m_{\pi} \leq n \operatorname{dim} \pi$ if $\pi \neq 1$. 
The Leopoldt conjecture for $H$ at the prime $p$ is equivalent to the equality $m_{\pi}=$ $\sum_{j=1}^{n} \operatorname{dim} \pi^{\{-, j\}}$ for every nontrivial $\pi$ and $m_{1}=1$. We can use [12, proof of Theorem 1], which uses the Baker-Brumer theorem on the $\overline{\mathbb{Q}}$-linear independence of $p$-adic logarithms of algebraic numbers, to get:

Lemma 4. Using the notation above, $m_{\pi}<n \operatorname{dim} \pi$, if $\pi=1$ or if $\pi \neq 1$ and $\operatorname{dim} \pi^{\{+, j\}} \neq 0$ for some $j$.

Lemma 5. If the Leopoldt conjecture is true for $H$, then the dimension of the $\overline{\mathbb{Q}}_{p}$-vector space $H^{1}\left(F, a d^{0} \rho\right)$ is $2 n$.

Proof. We have $H^{1}\left(F, \operatorname{ad}^{0} \rho\right)=\left(\operatorname{Hom}\left(G_{H}, \overline{\mathbb{Q}}_{p}\right) \otimes_{\overline{\mathbb{Q}}} \operatorname{ad}^{0} \rho\right)^{G}$. Since each irreducible component summand of $\operatorname{ad}^{0} \rho$ is non-trivial, self-dual and occurs multiplicity one (see [4, Section 4]), it follows, from Schur's lemma, that $\operatorname{dim}\left(\operatorname{Hom}\left(G_{H}, \overline{\mathbb{Q}}_{p}\right) \otimes_{\overline{\mathbb{Q}}}\right.$ $\left.\operatorname{ad}^{0} \rho\right)^{G}=\sum m_{\pi}$, where $\pi$ runs over all irreducible summands of $\operatorname{ad}^{0} \rho$. As $\rho$ is odd, the eigenvalues of $\operatorname{ad}^{0} \rho\left(\tau_{j}\right)$ are $1,-1,-1$ for $j=1, \cdots, n$. Therefore, for every summand $\pi$ of $\operatorname{ad}^{0} \rho$, we have either $\operatorname{dim} \pi^{\{-, j\}}=\operatorname{dim} \pi$ or $\operatorname{dim} \pi^{\{-, j\}}=\operatorname{dim} \pi-1$ for $j=1, \cdots, n$. If the Leopoldt conjecture for $H$ is true, then $m_{\pi}=\sum_{j=1}^{n} \operatorname{dim} \pi^{\{-, j\}}$ for all $\pi \in \hat{G}$. Hence, it follows that $\operatorname{dim}\left(\operatorname{Hom}\left(G_{H}, \overline{\mathbb{Q}}_{p}\right) \otimes_{\overline{\mathbb{Q}}} \operatorname{ad}^{0} \rho\right)^{G}=\sum m_{\pi}=$ $\sum \sum_{j=1}^{n} \operatorname{dim} \pi^{\{-, j\}}=\sum_{j=1}^{n} \operatorname{dim}\left(\operatorname{ad}^{0} \rho\right)^{\{-, j\}}=2 n$, where the first sum is taken over all irreducible summands of $\operatorname{ad}^{0} \rho$. This finishes the proof of the lemma.

\section{Bounds ON THE Dimension OF TANGENT SPACES}

Proposition 1. Suppose the Leopoldt conjecture is true for F. Then we have the following inequalities:

(1) $n+1 \leq \operatorname{dim} t_{\mathcal{D}} \leq \operatorname{dim} t_{\mathcal{D}^{\prime}}+n+1$

(2) $1 \leq \operatorname{dim} t_{\mathcal{D}_{0}} \leq \operatorname{dim} t_{\mathcal{D}^{\prime}}+1$

Proof. Recall that, we have the following description of the tangent spaces:

$$
\begin{aligned}
& t_{\mathcal{D}^{\prime}}=\operatorname{ker}\left(H^{1}\left(F, \operatorname{ad}^{0} \rho\right) \stackrel{\left(\left(C_{i, *}\right),\left(D_{i, *}\right)\right)}{\longrightarrow} \oplus_{i=1}^{r} H^{1}\left(F_{\mathfrak{p}_{i}}, \overline{\mathbb{Q}}_{p}\left(\psi_{i}^{\prime \prime} / \psi_{i}^{\prime}\right)\right) \bigoplus \oplus_{i=1}^{r} H^{1}\left(I_{\mathfrak{p}_{i}}, \overline{\mathbb{Q}}_{p}\right)\right) \\
& t_{\mathcal{D}_{0}}=\operatorname{ker}\left(H^{1}(F, \operatorname{ad} \rho) \stackrel{\left(\left(C_{i, *}\right),\left(D_{i, *}\right)\right)}{\longrightarrow} \oplus_{i=1}^{r} H^{1}\left(F_{\mathfrak{p}_{i}}, \overline{\mathbb{Q}}_{p}\left(\psi_{i}^{\prime \prime} / \psi_{i}^{\prime}\right)\right) \bigoplus \bigoplus_{i=1}^{r} H^{1}\left(I_{\mathfrak{p}_{i}}, \overline{\mathbb{Q}}_{p}\right)\right) \\
& t_{\mathcal{D}}=\operatorname{ker}\left(H^{1}(F, \operatorname{ad} \rho) \stackrel{\left(C_{i, *}\right)}{\longrightarrow} \oplus_{i=1}^{r} H^{1}\left(F_{\mathfrak{p}_{i}}, \overline{\mathbb{Q}}_{p}\left(\psi_{i}^{\prime \prime} / \psi_{i}^{\prime}\right)\right)\right)
\end{aligned}
$$

We know that $\operatorname{ad} \rho=\operatorname{ad}^{0} \rho \oplus \overline{\mathbb{Q}}_{p}$ and $\operatorname{dim} H^{1}\left(F, \overline{\mathbb{Q}}_{p}\right)=1$ as we have assumed the Leopoldt conjecture to be true for $F$. Thus, it follows that $\operatorname{dim} H^{1}(F, \operatorname{ad} \rho)=$ $\operatorname{dim} H^{1}\left(F, \operatorname{ad}^{0} \rho\right)+1$ and hence, $\operatorname{dim} t_{\mathcal{D}_{0}} \leq \operatorname{dim} t_{\mathcal{D}}+1$. 
From Tate's local Euler characteristic formula, we get that $\operatorname{dim} H^{1}\left(F_{\mathfrak{p}_{i}}, \overline{\mathbb{Q}}_{p}\left(\psi_{i}^{\prime \prime} / \psi_{i}^{\prime}\right)\right)=$ $\left[F_{\mathfrak{p}_{i}}: \mathbb{Q}_{p}\right]=e_{i} f_{i}$, where $e_{i}$ is the index of ramification and $f_{i}$ is the inertial degree of $\mathfrak{p}_{i}$, for $i=1, \cdots, r$. From local class field theory it follows that the rank of the restriction morphism $H^{1}\left(F_{\mathfrak{p}_{i}}, \overline{\mathbb{Q}}_{p}\right) \rightarrow H^{1}\left(I_{\mathfrak{p}_{i}}, \overline{\mathbb{Q}}_{p}\right)$ is $e_{i} f_{i}$ for $i=1, \cdots, r$. Thus, it follows that the rank of both the maps $C_{i, *}$ and $D_{i, *}$ is at most $e_{i} f_{i}$, for all $i$.

From the proof of Lemma 5 along with the discussion preceding the lemma, it follows that $\operatorname{dim} H^{1}\left(F, \operatorname{ad}^{0} \rho\right) \geq 2 n$. Since we assume that the Leopoldt conjecture is true for $F$, we get that $\operatorname{dim} H^{1}(F, \operatorname{ad} \rho)=1+\operatorname{dim} H^{1}\left(F, \operatorname{ad}^{0} \rho\right) \geq 2 n+1$. Hence, $\operatorname{dim} t_{\mathcal{D}_{0}}=\operatorname{dim} H^{1}(F, \operatorname{ad} \rho)-\sum_{i=1}^{r} \operatorname{rank}\left(C_{i, *}\right)-\sum_{i=1}^{r} \operatorname{rank}\left(D_{i, *}\right) \geq(2 n+1)-$ $\sum_{i=1}^{r} e_{i} f_{i}-\sum_{i=1}^{r} e_{i} f_{i}=(2 n+1)-n-n=1$. Similarly, $\operatorname{dim} t_{\mathcal{D}}=\operatorname{dim} H^{1}(F, \operatorname{ad} \rho)-$ $\sum_{i=1}^{r} \operatorname{rank}\left(C_{i, *}\right) \geq(2 n+1)-\sum_{i=1}^{r} e_{i} f_{i}=(2 n+1)-n=n+1$. To conclude the remaining inequality, it is enough to prove $\operatorname{dim} t_{\mathcal{D}}-n-1 \leq \operatorname{dim} t_{\mathcal{D}^{\prime}}$. Observe that $\operatorname{dim} t_{\mathcal{D}}-n-1=\operatorname{dim} t_{\mathcal{D}}-\sum_{i=1}^{r} e_{i} f_{i}-1=\operatorname{dim} H^{1}(F, \operatorname{ad} \rho)-\sum_{i=1}^{r} \operatorname{rank}\left(C_{i, *}\right)-$ $\sum_{i=1}^{r} e_{i} f_{i}-1 \leq\left(\operatorname{dim} H^{1}(F, \operatorname{ad} \rho)-1\right)-\sum_{i=1}^{r} \operatorname{rank}\left(C_{i, *}\right)-\sum_{i=1}^{r} \operatorname{rank}\left(D_{i, *}\right) \leq\left(\operatorname{dim} H^{1}\left(F, \operatorname{ad}^{0} \rho\right)\right)-$ $\sum_{i=1}^{r} \operatorname{rank}\left(C_{i, *}\right)-\sum_{i=1}^{r} \operatorname{rank}\left(D_{i, *}\right)=\operatorname{dim} t_{\mathcal{D}^{\prime}}$. Hence, it follows that $\operatorname{dim} t_{\mathcal{D}} \leq$ $\operatorname{dim} t_{\mathcal{D}^{\prime}}+n+1$ and the proof of the proposition is complete.

Fix an integer $i_{0}$ such that $1 \leq i_{0} \leq r$. Fix the basis $\left(e_{1, i_{0}}, e_{2, i_{0}}\right)$, which we introduced in section 1. So, under this basis, the image of $\left.\rho\right|_{G_{F_{\mathfrak{p}_{0}}}}$ is diagonal. Thus, from Lemma 3 , it follows that under this basis, an element of $t_{\mathcal{D}} \subset\left(\operatorname{Hom}\left(G_{H}, \overline{\mathbb{Q}}_{p}\right) \otimes\right.$ $\operatorname{ad} \rho)^{G}$ can be written as $\left(\begin{array}{ll}a & b \\ c & d\end{array}\right)$ with $a, b, c, d \in \operatorname{Hom}\left(G_{H}, \overline{\mathbb{Q}}_{p}\right)$. Recall that, we also have $\operatorname{Hom}\left(G_{H}, \overline{\mathbb{Q}}_{p}\right) \hookrightarrow \operatorname{Hom}\left(\left(\mathcal{O}_{H} \otimes_{\mathbb{Z}} \mathbb{Z}_{p}\right)^{\times}, \overline{\mathbb{Q}}_{p}\right) \simeq \oplus_{j=1}^{n} \overline{\mathbb{Q}}_{p}[G]$. Thus, we can see $a, b, c, d$ as elements $\sum_{j=1}^{n} \sum_{g \in G} a_{g, j} g, \sum_{j=1}^{n} \sum_{g \in G} b_{g, j} g, \sum_{j=1}^{n} \sum_{g \in G} c_{g, j} g$, $\sum_{j=1}^{n} \sum_{g \in G} d_{g, j} g$ of $\oplus_{j=1}^{n} \overline{\mathbb{Q}}_{p}[G]$ such that for every $g \in G$ and $1 \leq j \leq n$, we have:

$$
\left(\begin{array}{cc}
a_{g, j} & b_{g, j} \\
c_{g, j} & d_{g, j}
\end{array}\right)=\rho(g)\left(\begin{array}{cc}
a_{1, j} & b_{1, j} \\
c_{1, j} & d_{1, j}
\end{array}\right) \rho(g)^{-1}
$$

(Recall that, we are abusing the notation by denoting the image of $Y \in M_{2}\left(\overline{\mathbb{Q}}_{p}\right)$ by the adjoint action of $\operatorname{Proj}(\rho)(g)$ by $\left.\rho(g) Y \rho(g)^{-1}\right)$.

Proposition 2. The tangent space $t_{\mathcal{D}^{\prime}}$ has dimension at most $n$.

Proof. Let $\left(\begin{array}{ll}a & b \\ c & d\end{array}\right)$ be an element of $t_{\mathcal{D}^{\prime}}$. By Lemma 3, we have $c_{(i)}\left(G_{H_{w_{i}}}\right)=0$ and $d_{(i)}\left(I_{w_{i}}\right)=0$. We can view the elements $a_{(i)}, b_{(i)}, c_{(i)}, d_{(i)}$ as elements of the sum of $n$ copies of the group algebra. We will adapt the same notation as the one used for $a, b, c, d$ above for these elements. Note that, as we have fixed the basis $\left(e_{1, i_{0}}, e_{2, i_{0}}\right)$, $c_{i_{0}}=c$ and $d_{i_{0}}=d$. So, the above notation implies that $c_{1, j}=d_{1, j}=0$ for all $j$ such 
that the corresponding $\overline{\mathbb{Q}}$-embedding $i_{j}^{\prime}$ of $H$ lie in $J_{w_{0}}$, where $J_{w_{i_{0}}}$ is the subset of the set of all $\overline{\mathbb{Q}}$-embeddings of $H$ as defined in (1).

Let us recall the recipe of $c_{(i)}$ and $d_{(i)}$ : If $P_{i}$ is the change of basis matrix from $\left(e_{1, i_{0}}, e_{2, i_{0}}\right)$ to $\left(e_{1, i}, e_{2, i}\right)$, then $\left(\begin{array}{ll}a_{(i)} & b_{(i)} \\ c_{(i)} & d_{(i)}\end{array}\right)=P_{i}\left(\begin{array}{ll}a & b \\ c & d\end{array}\right) P_{i}^{-1}$. Thus, $\left(\begin{array}{cc}\left(a_{(i)}\right)_{g, k} & \left(b_{(i)}\right)_{g, k} \\ \left(c_{(i)}\right)_{g, k} & \left(d_{(i)}\right)_{g, k}\end{array}\right)=$ $P_{i}\left(\begin{array}{ll}a_{g, k} & b_{g, k} \\ c_{g, k} & d_{g, k}\end{array}\right) P_{i}^{-1}$ for all $1 \leq k \leq n$ and $g \in G$.

Now, the condition $c_{(i)}\left(G_{H_{w_{i}}}\right)=0$ and $d_{(i)}\left(I_{w_{i}}\right)=0$ implies that, if $i_{k}^{\prime} \in J_{w_{i}}$, then $\left(c_{(i)}\right)_{1, k}=\left(d_{(i)}\right)_{1, k}=0$. Since $a=-d$, we see that if $i_{k}^{\prime} \in J_{w_{i}}$, then $\left(\begin{array}{ll}\left(a_{(i)}\right)_{1, k} & \left(b_{(i)}\right)_{1, k} \\ \left(c_{(i)}\right)_{1, k} & \left(d_{(i)}\right)_{1, k}\end{array}\right)=\left(\begin{array}{cc}0 & \left(b_{(i)}\right)_{1, k} \\ 0 & 0\end{array}\right)$. Therefore, $\left(\begin{array}{ll}a_{1, k} & b_{1, k} \\ c_{1, k} & d_{1, k}\end{array}\right)=P_{i}^{-1}\left(\begin{array}{cc}0 & \left(b_{(i)}\right)_{1, k} \\ 0 & 0\end{array}\right) P_{i}$ and hence, $\left(\begin{array}{ll}a_{g, k} & b_{g, k} \\ c_{g, k} & d_{g, k}\end{array}\right)=\rho(g)\left(P_{i}^{-1}\left(\begin{array}{cc}0 & \left(b_{(i)}\right)_{1, k} \\ 0 & 0\end{array}\right) P_{i}\right) \rho(g)^{-1}$. We had chosen $i_{1}^{\prime}, \cdots, i_{n}^{\prime}$ such that, in the partition of the set of $\overline{\mathbb{Q}}$-embeddings of $H$ given in (1), $\left\{i_{1}^{\prime}, \cdots, i_{n}^{\prime}\right\} \subset \sqcup_{w_{i}} J_{w_{i}}$. Let us call $\left(b_{(i)}\right)_{1, k}$ as $b_{k}$ instead to simplify the notation. So, by combining all the observations above, we see that

$$
\sum_{k=1}^{n} \sum_{g \in G}\left(\begin{array}{ll}
a_{g, k} & b_{g, k} \\
c_{g, k} & d_{g, k}
\end{array}\right) g=\sum_{i=1}^{r} \sum_{k \in J_{w_{i}}} \sum_{g \in G}\left(\rho(g)\left(P_{i}^{-1}\left(\begin{array}{cc}
0 & b_{k} \\
0 & 0
\end{array}\right) P_{i}\right) \rho(g)^{-1}\right) g
$$

Since the matrices $P_{i}$ and $\rho(g)$ are fixed (because we have fixed a basis for each prime), we see that the element $\left(\begin{array}{ll}a & b \\ c & d\end{array}\right)$ of $t_{\mathcal{D}^{\prime}}$ is determined completely by the $n$-tuple $\left(b_{k}\right)_{1 \leq k \leq n}$ as above. Hence, it implies that $\operatorname{dim} t_{\mathcal{D}^{\prime}} \leq n$.

Remark. Note that, in the proof above, the bases $\left(e_{1, i}, e_{2, i}\right)$ that we are choosing satisfy the property that under the basis $\left(e_{1, i}, e_{2, i}\right)$, the image of $\left.\rho\right|_{G_{F_{p_{i}}}}$ is diagonal and $\rho\left(G_{F}\right) \subset G L_{2}(\overline{\mathbb{Q}})$. Moreover, $\rho$ has finite image. So, we can choose the change of basis matrices $P_{i}$ for the bases as above such that $P_{i} \in G L_{2}(\overline{\mathbb{Q}})$ for all $i$.

It turns out that, in most cases, we can get a slightly better upper bound on the dimension of $t_{\mathcal{D}^{\prime}}$.

Proposition 3. If there does not exist a totally real field $K$ of degree 2 over $F$ such that $\left.\rho\right|_{G_{K}}$ is reducible, then the dimension of the tangent space $t_{\mathcal{D}^{\prime}}$ is at most $n-1$.

Proof. Suppose $\operatorname{dim} t_{\mathcal{D}^{\prime}}>n-1$. Then, from Proposition 2, it follows that $\operatorname{dim} t_{\mathcal{D}^{\prime}}=$ $n$. From the equation (2) in the proof of Proposition 2 above, we see that, after fixing a basis $\left(e_{1, i_{0}}, e_{2, i_{0}}\right), \operatorname{dim} t_{\mathcal{D}^{\prime}}$ is equal to the dimension of the subspace of $\left(\overline{\mathbb{Q}}_{p}\right)^{n}$ consisting of $n$-tuples $\left(b_{1}, \ldots, b_{n}\right)$ such that if we substitute them in the RHS of (2), we get a matrix with entries in $H^{1}\left(H, \overline{\mathbb{Q}}_{p}\right)$. Hence, the equality $\operatorname{dim} t_{\mathcal{D}^{\prime}}=n$ implies that the matrix $\left(\begin{array}{ll}\sum_{g \in G} a_{g, k} g & \sum_{g \in G} b_{g, k} g \\ \sum_{g \in G} c_{g, k} g & \sum_{g \in G} d_{g, k} g\end{array}\right)=\sum_{g \in G}\left(\rho(g)\left(P_{i}^{-1}\left(\begin{array}{cc}0 & 1 \\ 0 & 0\end{array}\right) P_{i}\right) \rho(g)^{-1}\right) g$ 
is an element of $t_{\mathcal{D}^{\prime}}$ and hence, its entries lie in $H^{1}\left(H, \overline{\mathbb{Q}}_{p}\right)$ for every $1 \leq k \leq n$. Recall that, the $i$ in the equation above is such that $i_{k}^{\prime} \in J_{w_{i}}$.

From the remark after Proposition 2, it follows that $P_{i} \in G L_{2}(\overline{\mathbb{Q}})$ for every $1 \leq i \leq r$. As $\rho(g) \in P G L_{2}(\overline{\mathbb{Q}})$ for all $g \in G$ under the chosen basis for every prime, we get that $a_{g, k}, b_{g, k}, c_{g, k}, d_{g, k} \in \overline{\mathbb{Q}}$ for all $g \in G$ and $k=1, \cdots, n$. So, we can view $\sum_{g \in G} a_{g, k} g, \sum_{g \in G} b_{g, k} g, \sum_{g \in G} c_{g, k} g, \sum_{g \in G} d_{g, k} g$ as elements of the group algebra $\overline{\mathbb{Q}}[G]$ for all $k$. Note that, we also have a structural homomorphism $\overline{\mathbb{Q}}[G] \rightarrow \operatorname{Hom}_{\overline{\mathbb{Q}}}\left(\mathcal{O}_{H}^{\times} \otimes_{\mathbb{Z}} \overline{\mathbb{Q}}, \mathcal{O}_{H}^{\times} \otimes_{\mathbb{Z}} \overline{\mathbb{Q}}\right), g \in G \mapsto\left(u \otimes v \mapsto g^{-1}(u) \otimes v\right)$. Moreover, if we give $\operatorname{Hom}_{\overline{\mathbb{Q}}}\left(\mathcal{O}_{H}^{\times} \otimes_{\mathbb{Z}} \overline{\mathbb{Q}}, \mathcal{O}_{H}^{\times} \otimes_{\mathbb{Z}} \overline{\mathbb{Q}}\right)$ a structure of two-sided $G$-module in the following way: for $g_{1}, g_{2} \in G$ and $\phi \in \operatorname{Hom}_{\overline{\mathbb{Q}}}\left(\mathcal{O}_{H}^{\times} \otimes_{\mathbb{Z}} \overline{\mathbb{Q}}, \mathcal{O}_{H}^{\times} \otimes_{\mathbb{Z}} \overline{\mathbb{Q}}\right)$, then $\left(g_{1} \cdot \phi \cdot g_{2}\right)(u \otimes v)=$ $g_{2}^{-1}\left(\phi\left(g_{1}^{-1}(u) \otimes v\right)\right)$, then the map above is a map of $G$-bimodules i.e. it is equivariant for both right and left action of $G$ on both the sides.

It follows, by the argument used in the proof of [4, Theorem 3.5], that as $\sum_{g \in G} a_{g, k} g, \sum_{g \in G} b_{g, k} g, \sum_{g \in G} c_{g, k} g, \sum_{g \in G} d_{g, k} g$ are elements of both $H^{1}\left(H, \overline{\mathbb{Q}}_{p}\right)$ and $\overline{\mathbb{Q}}[G]$, they lie in the kernel of the structural homomorphism above. However, from the representation theory of finite groups, we know that the kernel of the structural map is isomorphic to $\oplus_{\pi} \pi^{\operatorname{dim} \pi}$, where the sum is over all $\pi \in \hat{G}$ which do not appear in $\mathcal{O}_{H}^{\times} \otimes_{\mathbb{Z}} \overline{\mathbb{Q}}$ (see [4, proof of Thm. 3.5]). From the structure of $\mathcal{O}_{H}^{\times} \otimes_{\mathbb{Z}} \overline{\mathbb{Q}}$, as a $G$-representation (which we know due to Minkowski's proof of Dirichlet's unit theorem and the Baker-Brumer theorem), we see that the kernel is isomorphic to $\oplus_{\pi} \pi^{\operatorname{dim} \pi}$, where $\pi \in \hat{G}$ is such that the only eigenvalue of $\pi\left(\tau_{i}\right)$ is -1 for $i=1, \cdots, n$.

Our assumptions on $\rho$ imply that we are in one of the following cases (see [4] for more details):

(1) $G$ is isomorphic to either $A_{4}, S_{4}$ or $A_{5}$ and $\operatorname{ad}^{0} \rho$ is irreducible.

(2) $G$ is a non-abelian dihedral group. In this case, there exists a unique quadratic extension $K$ of $F$ which is not totally real and a finite order character $\chi: G_{K} \rightarrow \overline{\mathbb{Q}}^{\times}$such that $\operatorname{ad}^{0} \rho \simeq \epsilon_{K} \oplus \operatorname{Ind}_{G_{K}}^{G_{F}}\left(\chi / \chi^{\sigma}\right)$ (we are using the same notations as used in the introduction). Thus, $\operatorname{ad}^{0} \rho$ is a sum of two irreducible representations.

(3) $G$ is isomorphic to $\mathbb{Z} / 2 \mathbb{Z} \times \mathbb{Z} / 2 \mathbb{Z}$. In this case, there exist three quadratic extensions $K, K^{\prime}$ and $K^{\prime \prime}$ of $F$ such that none of them is totally real and $\operatorname{ad}^{0} \rho \simeq \epsilon_{K} \oplus \epsilon_{K^{\prime}} \oplus \epsilon_{K^{\prime \prime}}$

Note that, the subspace $W$ of $\overline{\mathbb{Q}}[G]$ spanned by $\sum_{g \in G} a_{g, k} g, \sum_{g \in G} b_{g, k} g, \sum_{g \in G} c_{g, k} g$, $\sum_{g \in G} d_{g, k} g$ is $G$-stable and, as a $G$-representation, is isomorphic to a sub-representation 
of $\operatorname{ad}^{0} \rho$. Moreover, $W$ is non-trivial as at least one of its generators is non-zero, and is contained in the kernel of the structure homomorphism.

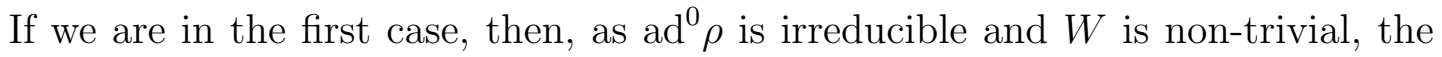
representation $W$ should be isomorphic $\operatorname{ad}^{0} \rho$. However, the eigenvalues of $\operatorname{ad}^{0} \rho\left(\tau_{j}\right)$ are $1,-1$ and -1 for all $\tau_{j}$ 's. Hence, the kernel of the structure homomorphism does not contain any copies of $\operatorname{ad}^{0} \rho$. Thus, we get a contradiction in this case.

If we are in the second case, then, as $K$ is not totally real, there exists a $\tau_{j}$ such that $\epsilon_{K}\left(\tau_{j}\right)=-1$. Thus, we see that the eigenvalues of $\operatorname{Ind}_{G_{K}}^{G_{F}}\left(\chi / \chi^{\sigma}\right)\left(\tau_{j}\right)$ are 1 and -1 and hence, the kernel of the structure homomorphism does not contain any copy of it. If $K$ is not CM, then there exists a $\tau_{j^{\prime}}$ such that $\epsilon_{K}\left(\tau_{j^{\prime}}\right)=1$. So, the kernel does not contain any copy of $\epsilon_{K}$ as well. Thus, $W$ should be 0 in this case. But we know that $W$ is non-zero. Therefore, we get a contradiction. If $K$ is a CM field, then $\epsilon_{K}\left(\tau_{j}\right)=-1$ for all $j$ and the eigenvalues of $\operatorname{Ind}_{G_{K}}^{G_{F}}\left(\chi / \chi^{\sigma}\right)\left(\tau_{j}\right)$ are 1 and -1 for every $j$. So, in this case, $W \simeq \epsilon_{K}$ and hence, it is a one dimensional vector space is generated by the non-zero vector $\sum_{g \in G} b_{g, k} g$. Since $a_{1, k}=c_{1, k}=d_{1, k}=0$ and $b_{1, k} \neq 0$, it follows that $\sum_{g \in G} a_{g, k} g=\sum_{g \in G} c_{g, k} g=\sum_{g \in G} d_{g, k} g=0$. This would imply that the subspace $\overline{\mathbb{Q}}_{p} e_{1, i}$ of $\rho$ is $G$ stable (see [4, proof of Thm. 2.2] for more details). But this is not possible as $\rho$ is irreducible. Thus, we get a contradiction in this case.

If we are in the third case, then, as none of $K, K^{\prime}$ and $K^{\prime \prime}$ are totally real, there exists $\tau_{j}, \tau_{j^{\prime}}$ and $\tau_{j^{\prime \prime}}$ such that $\epsilon_{K}\left(\tau_{j}\right)=-1, \epsilon_{K^{\prime}}\left(\tau_{j^{\prime}}\right)=-1$ and $\epsilon_{K^{\prime \prime}}\left(\tau_{j^{\prime \prime}}\right)=-1$. Thus, the kernel of the structure homomorphism does not contain a copy of any of these 3 representations. So, $W$ should be 0 . But we know that $W$ is non-zero. Thus, we get a contradiction in this case also.

The analysis above shows that, under the assumptions of this proposition, we get a contradiction if we assume $\operatorname{dim} t_{\mathcal{D}^{\prime}}>n-1$. Hence, it is proved that $\operatorname{dim} t_{\mathcal{D}^{\prime}} \leq n-1$ under the assumptions of this proposition.

We will study the tangent space $t_{\mathcal{D}^{\prime}}$ in the remaining case i.e. when there exists a totally real field $K$ of degree 2 over $F$ such that $\left.\rho\right|_{G_{K}}$ is reducible in $\S 6$.

\section{Description of the tangent spaces in the Dihedral Case}

Now suppose that $\operatorname{Proj}(\rho)=G$ is a dihedral group. So, there exists a quadratic extension $K$ of $F$ and a character $\chi: G_{K} \rightarrow \overline{\mathbb{Q}}^{\times}$such that $\rho \simeq \operatorname{Ind}_{G_{K}}^{G_{F}} \chi$. If $G$ is non-abelian, then $K$ is a unique such quadratic extension of $F$. In this case, $\operatorname{ad}^{0} \rho \simeq$ $\epsilon_{K} \oplus \operatorname{Ind}_{G_{K}}^{G_{F}}\left(\chi / \chi^{\sigma}\right)$, where $\epsilon_{K}$ is the quadratic character of $K, \sigma$ is the non-trivial 
element of $\operatorname{Gal}(K / F)$ and $\chi^{\sigma}$ is the character of $G_{K}$ given by $\chi^{\sigma}(g)=\chi\left(\sigma^{-1} g \sigma\right)$. In this section, we keep the notations used in the preceding sections.

Fix a basis $\left(v_{1}, v_{2}\right)$ of $V$ such that $\rho\left(G_{F}\right) \subset G L_{2}(\overline{\mathbb{Q}}),\left.\rho\right|_{G_{K}}$ acts by the character $\chi$ on the subspace generated by $v_{1}$ and by the character $\chi^{\sigma}$ on the subspace generated by $v_{2}$. Thus such a basis is well-defined up to scaling. If $\mathfrak{p}_{i}$ is split in $K$, then we can take $\left(e_{1, i}, e_{2, i}\right)$ to be either $\left(v_{1}, v_{2}\right)$ or $\left(v_{2}, v_{1}\right)$ depending on whether $\left.\chi^{\sigma}\right|_{G_{F_{\mathfrak{i}}}}=\psi_{i}^{\prime \prime}$ or $\left.\chi\right|_{G_{F_{\mathfrak{p}_{i}}}}=\psi_{i}^{\prime \prime}$. If $\mathfrak{p}_{i}$ is inert or ramified in $K$, then its image in the dihedral group $G$ contains an element of order 2 which is not contained in the image of $G_{K}$ in $G$. Call this element $\sigma_{i}$. There exists an $\ell_{i} \in \overline{\mathbb{Q}}$ such that $\operatorname{Proj}(\rho(\sigma))=$ $\left(\begin{array}{ll}0 & 1 \\ \ell_{i} & 0\end{array}\right) \in P G L_{2}(\overline{\mathbb{Q}})$. Fix a squareroot $\sqrt{\ell_{i}}$ of $\ell_{i}$ in $\overline{\mathbb{Q}}$. So $\rho\left(\sigma_{i}\right)$ will be diagonal under the basis $\left(v_{1}+\sqrt{\ell_{i}} v_{2}, v_{1}-\sqrt{\ell_{i}} v_{2}\right)$ which means we can take $\left(e_{1, i}, e_{2, i}\right)$ to be either $\left(v_{1}+\sqrt{\ell_{i}} v_{2}, v_{1}-\sqrt{\ell_{i}} v_{2}\right)$ or $\left(v_{1}-\sqrt{\ell_{i}} v_{2}, v_{1}+\sqrt{\ell_{i}} v_{2}\right)$. So, we fix the squareroot $\sqrt{\ell_{i}}$ so that $\left(e_{1, i}, e_{2, i}\right)=\left(v_{1}+\sqrt{\ell_{i}} v_{2}, v_{1}-\sqrt{\ell_{i}} v_{2}\right)$.

If $\left(\begin{array}{ll}a & b \\ c & d\end{array}\right)$ is an element of $t_{\mathcal{D}^{\prime}}$ under this basis $\left(v_{1}, v_{2}\right)$, then by combining the observations above along with the previous section, we have:

$$
\begin{array}{r}
\sum_{k=1}^{n} \sum_{g \in G}\left(\begin{array}{ll}
a_{g, k} & b_{g, k} \\
c_{g, k} & d_{g, k}
\end{array}\right) g=\sum_{k \in I_{1}}\left(\rho(g)\left(\begin{array}{cc}
0 & b_{k} \\
0 & 0
\end{array}\right) \rho(g)^{-1}\right) g+\sum_{k^{\prime} \in I_{2}}\left(\rho(g)\left(\begin{array}{cc}
0 & 0 \\
b_{k^{\prime}} & 0
\end{array}\right) \rho(g)^{-1}\right) g \\
+\sum_{k^{\prime \prime} \in I^{\prime}}\left(\rho(g)\left(\begin{array}{cc}
\frac{b_{k^{\prime \prime}}}{2} & \frac{-b_{k^{\prime \prime}}}{2 \sqrt{\ell_{i\left(k^{\prime \prime}\right)}}} \\
\frac{b_{k^{\prime \prime}} \sqrt{\ell_{i\left(k^{\prime \prime}\right)}}}{2} & \frac{-b_{k^{\prime \prime}}}{2}
\end{array}\right) \rho(g)^{-1}\right) g
\end{array}
$$

where $I_{1}$ is the subset of $\{1, \cdots, n\}$ such that if $k \in I$, then $i_{k}^{\prime} \in J_{w_{i}}$ with the prime $\mathfrak{p}_{i}$ of $F$ below $w_{i}$ is split in $K$ and $\left.\chi^{\sigma}\right|_{G_{F_{p_{i}}}}=\psi_{i}^{\prime \prime}, I_{2}$ is the subset of $\{1, \cdots, n\}$ such that if $k \in I$, then $i_{k}^{\prime} \in J_{w_{i}}$ with the prime $\mathfrak{p}_{i}$ of $F$ below $w_{i}$ is split in $K$ and $\left.\chi\right|_{G_{F_{\mathfrak{p}_{i}}}}=\psi_{i}^{\prime \prime}, I^{\prime}$ is the subset of $\{1, \cdots, n\}$ such that if $k^{\prime \prime} \in I^{\prime}$, then $i_{k^{\prime \prime}}^{\prime \prime} \in J_{w_{i}}$ with the prime of $F$ below $w_{i}$ is either inert or ramified in $K$ and for $k^{\prime \prime} \in I^{\prime}$, $i\left(k^{\prime \prime}\right)$ is such that if $i_{k^{\prime \prime}}^{\prime} \in J_{w_{i}}$ then the prime of $F$ below $w_{i}$ is $\mathfrak{p}_{i\left(k^{\prime \prime}\right)}$. Observe that $\{1, \cdots, n\}=I_{1} \sqcup I_{2} \sqcup I^{\prime}$.

Let $C$ be the image of $G_{K}$ in $G$ and let $\sigma^{\prime}$ be the element of $G$ such that its image in $P G L_{2}(\overline{\mathbb{Q}})$ is $\left(\begin{array}{ll}0 & 1 \\ 1 & 0\end{array}\right)$ under the basis $\left(v_{1}, v_{2}\right)$. Thus, from above, we get the following information:

(1) If $k \in I_{1} \sqcup I_{2}$, then $a_{g, k}=0$. If $k \in I^{\prime}$, then $a_{g, k}=\frac{b_{k}}{2}$ if $g \in C$ and $a_{g, k}=\frac{-b_{k}}{2}$ if $g \in G \backslash C$. 
(2) If $k \in I_{1}$, then $b_{g, k}=\left(\chi / \chi^{\sigma}\right)(g) b_{k}$ if $g \in C$ and $b_{g, k}=0$ if $g \in G \backslash C$. If $k \in I_{2}$, then $b_{g, k}=\left(\chi / \chi^{\sigma}\right)\left(g \sigma^{\prime}\right) b_{k}$ if $g \in G \backslash C$ and $b_{g, k}=0$ if $g \in C$. If $k \in I^{\prime}$, then $b_{g, k}=\left(\chi / \chi^{\sigma}\right)(g) \frac{-b_{k}}{2 \sqrt{\ell_{i}}}$ if $g \in C$ and $b_{g, k}=\left(\chi^{\sigma} / \chi\right)\left(\sigma^{\prime} g\right) \frac{b_{k} \sqrt{\ell_{i}}}{2}$ if $g \in G \backslash C$.

(3) If $k \in I_{1}$, then $c_{g, k}=\left(\chi^{\sigma} / \chi\right)\left(g \sigma^{\prime}\right) b_{k}$ if $g \in G \backslash C$ and $c_{g, k}=0$ if $g \in C$. If $k \in I_{2}$, then $c_{g, k}=\left(\chi^{\sigma} / \chi\right)(g) b_{k}$ if $g \in C$ and $c_{g, k}=0$ if $g \in G \backslash C$. If $k \in I^{\prime}$, then $c_{g, k}=\left(\chi^{\sigma} / \chi\right)(g) \frac{b_{k} \sqrt{\ell_{i}}}{2}$ if $g \in C$ and $c_{g, k}=\left(\chi / \chi^{\sigma}\right)\left(\sigma^{\prime} g\right) \frac{-b_{k}}{2 \sqrt{\ell_{i}}}$ if $g \in G \backslash C$.

(4) As $a=-d$, if $k \in I_{1} \sqcup I_{2}$, then $d_{g, k}=0$. If $k \in I^{\prime}$, then $d_{g, k}=\frac{-b_{k}}{2}$ if $g \in C$ and $d_{g, k}=\frac{b_{k}}{2}$ if $g \in G \backslash C$.

Using the same logic that we used in the case of $t_{\mathcal{D}^{\prime}}$, we see that if $\left(\begin{array}{ll}a & b \\ c & d\end{array}\right)$ is an element of $t_{\mathcal{D}_{0}}$ under the basis $\left(v_{1}, v_{2}\right)$, then

(4)

$$
\begin{array}{r}
\sum_{k=1}^{n} \sum_{g \in G}\left(\begin{array}{ll}
a_{g, k} & b_{g, k} \\
c_{g, k} & d_{g, k}
\end{array}\right) g=\sum_{k \in I_{1}}\left(\rho(g)\left(\begin{array}{cc}
a_{k} & b_{k} \\
0 & 0
\end{array}\right) \rho(g)^{-1}\right) g+\sum_{k^{\prime} \in I_{2}}\left(\rho(g)\left(\begin{array}{cc}
0 & 0 \\
b_{k^{\prime}} & a_{k^{\prime}}
\end{array}\right) \rho(g)^{-1}\right) g \\
+\sum_{k^{\prime \prime} \in I^{\prime}}\left(\rho(g)\left(\begin{array}{cc}
\frac{a_{k^{\prime \prime}}+b_{k^{\prime \prime}}}{2} & \frac{a_{k^{\prime \prime}}-b_{k^{\prime \prime}}}{2 \sqrt{\ell_{\left(k^{\prime \prime}\right)}}} \\
\frac{\left(a_{k^{\prime \prime}}+b_{k^{\prime \prime}}\right) \sqrt{\ell_{i\left(k^{\prime \prime}\right)}}}{2} & \frac{a_{k^{\prime \prime}}-b_{k^{\prime \prime}}}{2}
\end{array}\right) \rho(g)^{-1}\right) g
\end{array}
$$

and if $\left(\begin{array}{ll}a & b \\ c & d\end{array}\right)$ is an element of $t_{\mathcal{D}}$ under the basis $\left(v_{1}, v_{2}\right)$, then

$$
\begin{array}{r}
\sum_{k=1}^{n} \sum_{g \in G}\left(\begin{array}{ll}
a_{g, k} g & b_{g, k} g \\
c_{g, k} g & d_{g, k} g
\end{array}\right)=\sum_{k \in I_{1}} \rho(g)\left(\begin{array}{cc}
a_{k} g & b_{k} g \\
0 g & d_{k} g
\end{array}\right) \rho(g)^{-1}+\sum_{k^{\prime} \in I_{2}} \rho(g)\left(\begin{array}{cc}
d_{k^{\prime}} g & 0 g \\
b_{k^{\prime}} g & a_{k^{\prime}} g
\end{array}\right) \rho(g)^{-1} \\
+\sum_{k^{\prime \prime} \in I^{\prime}} \rho(g)\left(\begin{array}{cc}
\frac{a_{k^{\prime \prime}}+b_{k^{\prime \prime}}+d_{k^{\prime \prime}}}{2} g & \frac{a_{k^{\prime \prime}}-b_{k^{\prime \prime}}-d_{k^{\prime \prime}}}{2 \sqrt{\ell_{i\left(k^{\prime \prime}\right)}}} g \\
\frac{\left(a_{k^{\prime \prime}}+b_{k^{\prime \prime}}-d_{k^{\prime \prime}}\right) \sqrt{\ell_{i\left(k^{\prime \prime}\right)}}}{2} g & \frac{a_{k^{\prime \prime}}+d_{k^{\prime \prime}}-b_{k^{\prime \prime}}}{2} g
\end{array}\right) \rho(g)^{-1}
\end{array}
$$

Thus, we have a complete description of a possible elements of $t_{\mathcal{D}^{\prime}}, t_{\mathcal{D}_{0}}$ and $t_{\mathcal{D}}$ in the dihedral case. However, we still need to determine what values of $\left(a_{j}\right)_{1 \leq j \leq n}$, $\left(b_{j}\right)_{1 \leq j \leq n}$ and $\left(d_{j}\right)_{1 \leq j \leq n}$ will actually give an element of $t_{\mathcal{D}}$. We will do this analysis in the next few sections in order to prove Theorem 1 .

Observe that if $\left(\begin{array}{ll}a & b \\ c & d\end{array}\right)$ is an element of $t_{\mathcal{D}}$ under the basis $\left(v_{1}, v_{2}\right)$ chosen above, then the subspace of $\operatorname{Hom}\left(G_{H}, \overline{\mathbb{Q}}_{p}\right)$ generated by $a$ and $d$ is isomorphic to $1 \oplus \epsilon_{K}$ as a $G$-representation. To be more precise, the subspace generated by $a+d$ is isomorphic to 1 and the subspace generated by $a-d$ is isomorphic to $\epsilon_{K}$ as $G$ representations. The subspace generated by $b$ and $c$ is isomorphic to $\operatorname{Ind}_{G_{K}}^{G_{F}}\left(\chi / \chi^{\sigma}\right)$ as a $G$-representation. If the Leopoldt conjecture is true for $F$, then it implies that the trivial representation 1 occurs in $\operatorname{Hom}\left(G_{H}, \overline{\mathbb{Q}}_{p}\right)$ with multiplicity one and it is generated by the element $\sum_{k=1}^{n} \sum_{g \in G} g$ of $\oplus_{k=1}^{n} \overline{\mathbb{Q}}_{p}[G]$. Thus, this implies that if 
$\left(\begin{array}{ll}a & b \\ c & d\end{array}\right)$ is an element of $t_{\mathcal{D}}$ under the basis $\left(v_{1}, v_{2}\right)$ and if the Leopoldt conjecture is true for $F$, then $a_{1}+d_{1}=\cdots=a_{k}+d_{k}=\cdots=a_{n}+d_{n}$, where $a_{k}$ and $d_{k}$ are defined as in the last equation above. We will find more relations in this spirit in the next few sections to prove Theorem 1 .

\section{Proof of Theorem 1 for CASES 1 And 2}

Suppose that $\rho \simeq \operatorname{Ind}_{G_{K}}^{G_{F}} \chi$ and $K$ is totally real i.e. it has $2 n$ real embeddings. Here, we do not put the assumption that $G$ is non-abelian. So, it can be $\mathbb{Z} / 2 \mathbb{Z} \times$ $\mathbb{Z} / 2 \mathbb{Z}$. Recall that, in this case, $\operatorname{ad}^{0} \rho=\epsilon_{K} \oplus \operatorname{Ind}_{G_{K}}^{G_{F}}\left(\chi / \chi^{\sigma}\right)$. Fix the basis $\left(v_{1}, v_{2}\right)$ as in the previous section and we will also use the notation of the last section. Let $C$ be the image of $G_{K}$ in $G$. As $K$ is totally real, $G$ is a dihedral group with order divisible by 4 and $\tau_{1}=\cdots=\tau_{n}=\tau$, where $\tau$ is the unique element of $C$ of order 2 .

As $\epsilon_{K}(\tau)=1$ and $K$ is totally real, $\epsilon_{K}$ appears in $\operatorname{Hom}\left(\mathcal{O}_{H}^{\times}, \overline{\mathbb{Q}}_{p}\right)$ with multiplicity $n$. Thus, if the Leopoldt conjecture is true for $K$, then $\epsilon_{K}$ does not appear at all in $\operatorname{Hom}\left(G_{H}, \overline{\mathbb{Q}}_{p}\right)$. On the other hand, $\operatorname{Ind}_{G_{K}}^{G_{F}}\left(\chi / \chi^{\sigma}\right)$ appears with full multiplicity in $\operatorname{Hom}\left(G_{H}, \overline{\mathbb{Q}}_{p}\right)$ i.e. it appears with multiplicity $2 n$ because the eigenvalues of $\operatorname{Ind}_{G_{K}}^{G_{F}}\left(\chi / \chi^{\sigma}\right)(\tau)$ are -1 and -1 .

Suppose the Leopoldt conjecture is true for $K$. Hence, it is true for $F$ as well. If $\left(\begin{array}{ll}a & b \\ c & d\end{array}\right) \in t_{\mathcal{D}}$, then from the last section, we see that $a-d$ generates a representation isomorphic to $\epsilon_{K}$. As it does not appear in $\operatorname{Hom}\left(G_{H}, \overline{\mathbb{Q}}_{p}\right), a-d$ should be 0 . Thus, from (5), we have $a_{k}=d_{k}$ if $k \in I_{1} \sqcup I_{2}$ and $b_{k}=0$ if $k \in I^{\prime}$. Moreover, as the Leopoldt conjecture is true for $F, a_{1}+d_{1}=\cdots=a_{n}+d_{n}$ and it follows from (5) that $t_{\mathcal{D}}$ is generated by at most $n+1$ elements. However, by Proposition 1 . $\operatorname{dim} t_{\mathcal{D}} \geq n+1$, if the Leopoldt conjecture is true for $F$. Thus, $\operatorname{dim} t_{\mathcal{D}}=n+1$.

Recall that $t_{\mathcal{D}^{\prime}}$ is the subspace of $t_{\mathcal{D}}$ of elements $\left(\begin{array}{ll}a & b \\ c & d\end{array}\right)$ such that $c_{k}=d_{k}=$ $a_{k}=0$ for all $k$. From the last paragraph, we know the generators of $t_{\mathcal{D}}$ explicitly. They are obtained from all the $3 n$-tuples $\left(\left(a_{k}\right),\left(b_{k}\right),\left(d_{k}\right)\right)$ satisfying the relations $a_{k}=d_{k}$ if $k \in I_{1} \sqcup I_{2}, b_{k}=0$ if $k \in I^{\prime}$ and $a_{1}+d_{1}=\cdots=a_{n}+d_{n}$ as above. Combining them, with the relations $a_{k}=d_{k}=0$, we see that the generators are obtained from all the $3 n$-tuples $\left(\left(a_{k}\right),\left(b_{k}\right),\left(d_{k}\right)\right)$ such that $a_{k}=d_{k}=0$ for all $k$ and $b_{k}=0$ for $k \in I^{\prime}$. Hence, borrowing the notation from introduction, we see that $\operatorname{dim} t_{\mathcal{D}^{\prime}}=\left|I_{1}\right|+\left|I_{2}\right|=\sum_{\mathfrak{p}_{i} \in S} e_{i} f_{i}$.

Note that, as $\operatorname{Ind}_{G_{K}}^{G_{F}}\left(\chi / \chi^{\sigma}\right)$ appears with full multiplicity in $\operatorname{Hom}\left(G_{H}, \overline{\mathbb{Q}}_{p}\right)$, we get the inequality $\operatorname{dim} t_{\mathcal{D}^{\prime}} \geq\left|I_{1}\right|+\left|I_{2}\right|$ from the elements obtained above without 
the Leopoldt conjecture for $K$. We need the Leopoldt conjecture for $K$ to establish the equality. Thus, if all primes of $F$ lying above $p$ are split in $K$, then we get $\operatorname{dim} t_{\mathcal{D}^{\prime}}=n$ without the Leopoldt conjecture for $K$. Suppose there exists at least one prime of $F$ lying above $p$ which is not split in $K$. If, in this case $\operatorname{dim} t_{\mathcal{D}^{\prime}}=n$, then we obtain, from the description of elements of $t_{\mathcal{D}^{\prime}}$ found in the previous section, elements of $\operatorname{Hom}\left(G_{H}, \overline{\mathbb{Q}}_{p}\right)$ belonging to the isotypic component of $\epsilon_{K}$ which are also elements of $\overline{\mathbb{Q}}[G]$. The same argument that was used in the proof of Proposition 3 would give us a contradiction in this case. Hence, it follows that, in this case, $\operatorname{dim} t_{\mathcal{D}^{\prime}} \leq n-1$ without the Leopoldt conjecture. Thus, we see that the results similar to that of Proposition 3 also hold in the case excluded in the proposition.

If $\sum_{\mathfrak{p}_{i} \in S} e_{i} f_{i}=0$ i.e. $\operatorname{dim} t_{\mathcal{D}^{\prime}}=0$, then Proposition 1 implies that $\operatorname{dim} t_{\mathcal{D}_{0}}=1$. Suppose $\sum_{\mathfrak{p}_{i} \in S} e_{i} f_{i} \geq 1$, which means $\operatorname{dim} t_{\mathcal{D}^{\prime}} \geq 1$. Let $\left(\begin{array}{ll}a & b \\ c & d\end{array}\right)$ be an element of $t_{\mathcal{D}_{0}}$. Then, we know that $d_{1}=\cdots=d_{n}=0$ and hence, $a_{1}=\cdots=a_{n}$. As $a-d$ generates $\epsilon_{K}$, we should have $a=d$. Suppose $\mathfrak{p}_{j}$ be a prime of $F$ above $p$ which is split in $K$. Such a prime exists because $\sum_{\mathfrak{p}_{i} \in S} e_{i} f_{i} \geq 1$. So, $a=d$ implies that $a_{j}=d_{j}$. Hence, all $a_{i}$ 's are also 0 and $\left(\begin{array}{ll}a & b \\ c & d\end{array}\right) \in t_{\mathcal{D}^{\prime}}$. Thus, in this case, $\operatorname{dim} t_{\mathcal{D}^{\prime}}=\operatorname{dim} t_{\mathcal{D}_{0}}$. Therefore, $\operatorname{dim} t_{\mathcal{D}_{0}}=\max \left\{\sum_{\mathfrak{p}_{i} \in S} e_{i} f_{i}, 1\right\}$. This finishes the proof of the first case of the theorem.

Now suppose that $\rho \simeq \operatorname{Ind}_{G_{K}}^{G_{F}} \chi, G$ is non-abelian and $K$ has exactly $2(n-1)$ real embeddings. Fix the basis $\left(v_{1}, v_{2}\right)$ as in the previous section. Retaining the notations from the previous case, we see that $G$ is a non-abelian dihedral group with order divisible by 4 , (after reordering $\tau_{i}$ 's if necessary) $\tau_{1}=\cdots=\tau_{n-1}=\tau$ and $\tau_{n} \in G \backslash C$. If the Leopoldt conjecture is true for $K$, then $\epsilon_{K}$ appears in $\operatorname{Hom}\left(G_{H}, \overline{\mathbb{Q}}_{p}\right)$ with multiplicity 1 . On the other hand, from Lemma 4 and the discussion made just before the lemma, it follows that $\operatorname{Ind}_{G_{K}}^{G_{F}}\left(\chi / \chi^{\sigma}\right)$ appears with multiplicity $2 n-1$ in $\operatorname{Hom}\left(G_{H}, \overline{\mathbb{Q}}_{p}\right)$.

Suppose the Leopoldt conjecture is true for $K$ and hence, for $F$. Then, the trivial representation 1 appears in $\operatorname{Hom}\left(G_{H}, \overline{\mathbb{Q}}_{p}\right)$ with multiplicity one. Let $\left(\begin{array}{ll}a & b \\ c & d\end{array}\right)$ be an element of $t_{\mathcal{D}}$. We see from above that $a-d$ generates $\epsilon_{K}$ and $a+d$ generates 1 . Both of them occur in $\operatorname{Hom}\left(G_{H}, \overline{\mathbb{Q}}_{p}\right)$ with multiplicity 1 . So, if $W$ is the subspace of $t_{\mathcal{D}}$ such that $a+d=a-d=0$, then, from above it follows that $\operatorname{dim} t_{\mathcal{D}} \leq \operatorname{dim} W+2$. Thus, if $\left(\begin{array}{ll}a & b \\ c & d\end{array}\right) \in W$, then $a=d=0$ which implies that in $50, a_{k}=d_{k}=0$ if $k \in I_{1} \sqcup I_{2}, b_{k^{\prime}}=0$ and $a_{k^{\prime}}+d_{k^{\prime}}=0$ if $k^{\prime} \in I^{\prime}$. So, it follows that $\operatorname{dim} W \leq n$. Note that, $W$ is just a direct sum of some copies of $\operatorname{Ind}\left(\chi / \chi^{\sigma}\right)$. 
It follows, from (5) and the discussion above, that a general element of $W$ under the chosen basis is of the form $\sum_{k=1}^{n} \sum_{g \in G}\left(\rho(g)\left(\begin{array}{cc}0 & b_{k} \\ c_{k} & 0\end{array}\right) \rho(g)^{-1}\right) g$ with the conditions $c_{k}=0$ if $k \in I_{1}, b_{k}=0$ if $k \in I_{2}$ and $\ell_{i\left(k^{\prime}\right)} b_{k^{\prime}}=c_{k^{\prime}}$ if $k^{\prime} \in I^{\prime}$. So, $W$ is a subspace of the isotypic component of $\operatorname{Hom}\left(G_{H}, \overline{\mathbb{Q}}_{p}\right)$ corresponding to $\operatorname{Ind}\left(\chi / \chi^{\sigma}\right)$ defined by $n$ linear relations. As the dimension of the isotypic component of $\operatorname{Ind}\left(\chi / \chi^{\sigma}\right)$ in $\operatorname{Hom}\left(G_{H}, \overline{\mathbb{Q}}_{p}\right)$ is $2 n-1$, we get that $\operatorname{dim} W \geq 2 n-1-n=n-1$. If $\operatorname{dim} W=n$, then the description of a general element of $W$ found above gives us elements of $\operatorname{Hom}\left(G_{H}, \overline{\mathbb{Q}}_{p}\right)$ belonging to the isotypic component of $\operatorname{Ind}\left(\chi / \chi^{\sigma}\right)$ which are also elements of $\overline{\mathbb{Q}}[G]$. Since, $\operatorname{Ind}\left(\chi / \chi^{\sigma}\right)$ appears in $\operatorname{Hom}\left(\mathcal{O}_{H}^{\times}, \overline{\mathbb{Q}}_{p}\right)$, the same argument that was used in the proof of Proposition 3 would give us a contradiction and hence, imply that $\operatorname{dim} W<n$. Thus, $\operatorname{dim} W=n-1$ and $\operatorname{dim} t_{\mathcal{D}} \leq n+1$. By Proposition 1 , we have $\operatorname{dim} t_{\mathcal{D}} \geq n+1$. Hence, $\operatorname{dim} t_{\mathcal{D}}=n+1$.

Note that, as $t_{\mathcal{D}^{\prime}}$ is obtained from $t_{\mathcal{D}}$ by putting the extra conditions $a_{k}=d_{k}=0$ for all $k$ in (5), the subspace $t_{\mathcal{D}^{\prime}} \cap W$ of $W$ is obtained by putting the extra conditions $a_{k}=d_{k}=0$ for $k \in I^{\prime}$ on the elements of $W$. Thus, $\operatorname{dim} t_{\mathcal{D}^{\prime}} \geq \operatorname{dim}\left(t_{\mathcal{D}^{\prime}} \cap W\right) \geq$ $\operatorname{dim} W-\left|I^{\prime}\right|=n-1-\left|I^{\prime}\right|=\left|I_{1}\right|+\left|I_{2}\right|-1=\sum_{\mathfrak{p}_{i} \in S} e_{i} f_{i}-1$. By Proposition 3, we have $\operatorname{dim} t_{\mathcal{D}^{\prime}} \leq n-1$. This concludes the proof of Theorem 1 for case 2 .

Observe that, we did not use the Leopoldt conjecture to get this lower bound. Indeed, we only used the equality $\operatorname{dim} \operatorname{Hom}_{G}\left(\operatorname{Ind}_{G_{K}}^{G_{F}}\left(\chi / \chi^{\sigma}\right), \operatorname{Hom}\left(G_{H}, \overline{\mathbb{Q}}_{p}\right)\right)=2 n-1$ along with the descriptions of $W$ and $t_{\mathcal{D}^{\prime}}$, all of which are obtained without using the Leopoldt conjecture. If all primes of $F$ lying above $p$ are split in $K$ i.e. $\sum_{\mathfrak{p}_{i} \in S} e_{i} f_{i}=$ $n$, then we have proved above (without assuming the Leopoldt conjecture) that $\operatorname{dim} t_{\mathcal{D}^{\prime}} \geq n-1$. By Proposition 3, we have $\operatorname{dim} t_{\mathcal{D}^{\prime}} \leq n-1$. Hence, we get $\operatorname{dim} t_{\mathcal{D}^{\prime}}=n-1$ unconditionally if all primes of $F$ above $p$ are split in $K$.

\section{Proof of Theorem 1 for CASE 4}

Suppose $F$ is a real quadratic field. We are assuming that $\rho=\operatorname{Ind}_{G_{K}}^{G_{F}} \chi, G$ is a non-abelian dihedral group, $K$ has exactly 2 real embeddings and $\sum_{\mathfrak{p}_{i} \in S} e_{i} f_{i} \geq 1$. Therefore, $\operatorname{rank}_{\mathbb{Z}}\left(\mathcal{O}_{K}^{\times}\right)=2$ and $\operatorname{rank}_{\mathbb{Z}}\left(\mathcal{O}_{F}^{\times}\right)=1$. As $K$ is $G$-stable, $\mathcal{O}_{K}^{\times}$is also $G$ stable. As a $G$-representation, $\mathcal{O}_{K}^{\times} \otimes_{\mathbb{Z}} \overline{\mathbb{Q}} \simeq 1 \oplus \epsilon_{K}$. So, by [12, proof of Theorem 1], it follows that the Leopoldt conjecture is true for $K$.

If all primes of $F$ above $p$ are split in $K$, then, the observations made in the previous paragraph imply that we are in the special instance of the second case of Theorem 1 recorded in the last paragraph of $\S 6$ with $n=2$. Thus, it follows, from 
the second case of Theorem 1 , that $\operatorname{dim} t_{\mathcal{D}^{\prime}}=n-1=2-1=1$ and $\operatorname{dim} t_{\mathcal{D}}=$ $n+1=2+1=3$.

Fix the basis $\left(v_{1}, v_{2}\right)$ as in the previous section. Let $\left(\begin{array}{ll}a & b \\ c & d\end{array}\right)$ be an element of $t_{\mathcal{D}_{0}}$. As all primes of $F$ above $p$ are split in $K$, we see, from (4), that either

$$
\left(\begin{array}{ll}
a & b \\
c & d
\end{array}\right)=\sum_{k=1}^{2} \sum_{g \in G}\left(\rho(g)\left(\begin{array}{cc}
a_{k} & b_{k} \\
0 & 0
\end{array}\right) \rho(g)^{-1}\right) g
$$

or

$$
\left(\begin{array}{ll}
a & b \\
c & d
\end{array}\right)=\sum_{g \in G}\left(\rho(g)\left(\begin{array}{cc}
a_{1} & b_{1} \\
0 & 0
\end{array}\right) \rho(g)^{-1}\right) g+\sum_{g \in G}\left(\rho(g)\left(\begin{array}{cc}
0 & 0 \\
b_{2} & a_{2}
\end{array}\right) \rho(g)^{-1}\right) g
$$

In particular, we see that either $a=\sum_{k=1}^{2} a_{k} \sum_{g \in C} g$ or $a=a_{1} \sum_{g \in C} g+a_{2} \sum_{g \in C} g \sigma^{\prime}$ (note that $\sum_{g \in C}$ and $\sum_{g \in C} g \sigma^{\prime}$ belong to two different copies of the group algebra). As the Leopoldt conjecture is true for $F$, it follows that $a_{1}=a_{2}$. So, either $a=$ $a_{1}\left(\sum_{k=1}^{2} \sum_{g \in C} g\right)$ or $a=a_{1} \sum_{g \in C} g+a_{1} \sum_{g \in C} g \sigma^{\prime}$. Let $u$ be a unit of $K$ such that with $\mathcal{O}_{F}^{\times}$, it generates a finite index subgroup of $\mathcal{O}_{K}^{\times}$. Suppose $a=a_{1}\left(\sum_{k=1}^{2} \sum_{g \in C} g\right)$. As $a \in \operatorname{Hom}\left(G_{H}, \overline{\mathbb{Q}}_{p}\right), a(u)=0$ i.e. $a_{1}\left(\sum_{g \in C} \log _{p}\left(i_{p} \circ i_{1}^{\prime}\left(g^{-1} u\right)\right)+\sum_{g \in C} \log _{p}\left(i_{p} \circ\right.\right.$ $\left.\left.i_{2}^{\prime}\left(g^{-1} u\right)\right)\right)=0$. Since $C$ acts trivially on $K$, we have $a_{1}\left(\log _{p}\left(i_{p} \circ i_{1}^{\prime}(u)\right)+\log _{p}\left(i_{p} \circ\right.\right.$ $\left.\left.i_{2}^{\prime}(u)\right)\right)=0$ and hence, $a_{1}\left(\log _{p}\left(i_{p} \circ\left(i_{1}^{\prime}(u) i_{2}^{\prime}(u)\right)\right)\right)=0$.

As $K$ has exactly 2 real embeddings, our choice of $i_{1}^{\prime}$ and $i_{2}^{\prime}$ forces one of them to be a complex embedding and the other one to be a real embedding. Without loss of generality, suppose $i_{1}^{\prime}$ is a real embedding. Now if $\left(i_{1}^{\prime}(u) i_{2}^{\prime}(u)\right)^{n}=1$ for some $n$, then we see that $i_{2}^{\prime}\left(u^{n}\right)$ is real. But $i_{2}^{\prime}$ is a complex embedding of $K$ and the maximal real subfield of $i_{2}^{\prime}(K)$ is $i_{2}(F)$. So, we see that $i_{2}^{\prime}\left(u^{n}\right) \in i_{2}(F)$ which implies that $u^{n} \in F$. But this contradicts our assumption that $u$ generates a finite index subgroup of $\mathcal{O}_{K}^{\times}$along with $\mathcal{O}_{F}^{\times}$. So, $i_{1}^{\prime}(u) i_{2}^{\prime}(u)$ is not a root of unity which implies that $\log _{p}\left(i_{p} \circ\left(i_{1}^{\prime}(u) i_{2}^{\prime}(u)\right)\right) \neq 0$. Thus, we get that $a_{1}=0$. Therefore, $\left(\begin{array}{ll}a & b \\ c & d\end{array}\right) \in t_{\mathcal{D}^{\prime}}$ and hence, $t_{\mathcal{D}_{0}}=t_{\mathcal{D}^{\prime}}$.

Suppose $a=a_{1} \sum_{g \in C} g+a_{1} \sum_{g \in G \backslash C} g \sigma^{\prime}$. So, $a(u)=0$ means $a_{1}\left(\sum_{g \in C} \log _{p}\left(i_{p} \circ\right.\right.$ $\left.\left.i_{1}^{\prime}\left(g^{-1} u\right)\right)+\sum_{g \in C} \log _{p}\left(i_{p} \circ i_{2}^{\prime}\left(\sigma^{\prime} g^{-1} u\right)\right)\right)=0$. Since $C$ acts trivially on $\mathrm{K}$, we have $a_{1}\left(\log _{p}\left(i_{p} \circ\left(i_{1}^{\prime}(u) i_{2}^{\prime}\left(\sigma^{\prime}(u)\right)\right)\right)\right)=0$. As $K$ has exactly 2 real embeddings, our choice of $i_{1}^{\prime}$ and $i_{2}^{\prime}$ forces one of $i_{1}^{\prime}$ and $i_{2}^{\prime} \circ \sigma^{\prime}$ to be a complex embedding and the other one to be a real embedding. Therefore, by the same logic as in the previous paragraph, we get $a_{1}=0$ and $t_{\mathcal{D}_{0}}=t_{\mathcal{D}^{\prime}}$. Therefore, in both the cases, $\operatorname{dim} t_{\mathcal{D}_{0}}=\operatorname{dim} t_{\mathcal{D}^{\prime}}=1$.

Suppose that one of the primes of $F$ above $p$ splits in $K$ and the other remains inert or ramifies in $K$. Then, (without loss of generality) an element $\left(\begin{array}{ll}a & b \\ c & d\end{array}\right) \in t_{\mathcal{D}^{\prime}}$ 
looks like

$$
\left(\begin{array}{ll}
a & b \\
c & d
\end{array}\right)=\sum_{g \in G}\left(\rho(g)\left(\begin{array}{cc}
0 & b_{1} \\
0 & 0
\end{array}\right) \rho(g)^{-1}\right) g+\sum_{g \in G}\left(\rho(g)\left(\begin{array}{cc}
\frac{b_{2}}{2} & \frac{-b_{2}}{2 \sqrt{\ell_{i(2)}}} \\
\frac{b_{2} \sqrt{\ell_{i(2)}}}{2} & \frac{-b_{2}}{2}
\end{array}\right) \rho(g)^{-1}\right) g
$$

In particular, if $u \in \mathcal{O}_{H}^{\times}, a(u)=\frac{b_{2}}{2}\left(\sum_{g \in C} \log _{p}\left(i_{p} \circ i_{2}^{\prime}\left(g^{-1}(u)\right)\right)-\sum_{g \in G \backslash C} \log _{p}\left(i_{p} \circ\right.\right.$ $\left.\left.i_{2}^{\prime}\left(g^{-1}(u)\right)\right)\right)$.

Note that, $\sum_{g \in C} g-\sum_{g \in G \backslash C} g \in \overline{\mathbb{Q}}[G]$. The $\overline{\mathbb{Q}}$-subspace spanned by this element is $G$-stable and isomorphic to $\epsilon_{K}$ as a $G$-representation. But we know that $\epsilon_{K}$ appears in $\operatorname{Hom}\left(\mathcal{O}_{H}^{\times}, \overline{\mathbb{Q}}_{p}\right)$. Hence, from the proof of Proposition 3 and [4, proof of Theorem 3.5], we see that there exists a unit $u_{0} \in \mathcal{O}_{H}^{\times}$such that $\sum_{g \in C} \log _{p}\left(i_{p} \circ\right.$ $\left.i_{2}^{\prime}\left(g^{-1}\left(u_{0}\right)\right)\right)-\sum_{g \in G \backslash C} \log _{p}\left(i_{p} \circ i_{2}^{\prime}\left(g^{-1}\left(u_{0}\right)\right)\right) \neq 0$. But as $a \in \operatorname{Hom}\left(G_{H}, \overline{\mathbb{Q}}_{p}\right), a(u)=0$ for all $u \in \mathcal{O}_{H}^{\times}$. So, we get that $b_{2}=0$.

So, we have $\left(\begin{array}{ll}a & b \\ c & d\end{array}\right)=\sum_{g \in G}\left(\rho(g)\left(\begin{array}{cc}0 & b_{1} \\ 0 & 0\end{array}\right) \rho(g)^{-1}\right) g$. Thus, we see that $a=$ $d=0$ and $b, c$ generate a representation isomorphic to $\operatorname{Ind}_{G_{K}}^{G_{F}}\left(\chi / \chi^{\sigma}\right)$. Moreover, $b=b_{1}\left(b^{\prime}\right)$ and $c=b_{1}\left(c^{\prime}\right)$, where $b^{\prime}, c^{\prime} \in \overline{\mathbb{Q}}[G]$. We know that $\operatorname{Ind}_{G_{K}}^{G_{F}}\left(\chi / \chi^{\sigma}\right)$ appears in $\operatorname{Hom}\left(\mathcal{O}_{H}^{\times}, \overline{\mathbb{Q}}_{p}\right)$. So, from the proof of Proposition 3, we see that $b_{1}=0$. Hence, we get $\operatorname{dim} t_{\mathcal{D}^{\prime}}=0$. Therefore, from Proposition 11, it follows that $\operatorname{dim} t_{\mathcal{D}_{0}}=1$ and $\operatorname{dim} t_{\mathcal{D}}=3$. This concludes the proof of Theorem 1 for the fourth case.

\section{Proof of Theorem 1 for case 3}

Suppose $\rho=\operatorname{Ind}_{G_{K}}^{G_{F}} \chi, G$ is a non-abelian dihedral group, $K$ is a CM field and all primes of $F$ above $p$ are split in $K$. We retain the notations that we have been using so far. As $K$ is a CM field, $\tau_{i} \notin C$ for all $i$. Thus, $\operatorname{Ind}_{G_{K}}^{G_{F}}\left(\chi / \chi^{\sigma}\right)$ appears in $\mathcal{O}_{H}^{\times} \otimes_{\mathbb{Z}} \overline{\mathbb{Q}}$ with multiplicity $n$. Hence, the subspace $V_{\chi / \chi^{\sigma}}$ of $\mathcal{O}_{H}^{\times} \otimes_{\mathbb{Z}}$ $\overline{\mathbb{Q}}$ on which $C$ acts by $\chi / \chi^{\sigma}$ has dimension $n$. Let $x_{1}, \cdots, x_{n} \in \mathcal{O}_{H}^{\times}$be such that $\left\{\left(\sum_{g \in C}\left(\chi / \chi^{\sigma}\right)(g) g^{-1}\right)\left(x_{1} \otimes 1\right), \cdots,\left(\sum_{g \in C}\left(\chi / \chi^{\sigma}\right)(g) g^{-1}\right)\left(x_{n} \otimes 1\right)\right\}$ is a basis of $V_{\chi / \chi^{\sigma}}$ (it is clear that we can find such units). Therefore, if the Leopoldt conjecture is true for $H$, then $\operatorname{Ind}_{G_{K}}^{G_{F}}\left(\chi / \chi^{\sigma}\right)$ appears in $\operatorname{Hom}\left(G_{H}, \overline{\mathbb{Q}}_{p}\right)$ with multiplicity $n$.

Fix the basis $\left(v_{1}, v_{2}\right)$ as in the previous section. As all primes of $F$ above $p$ are split in $K$, from the formulas we found earlier, it follows that if $\left(\begin{array}{ll}a & b \\ c & d\end{array}\right) \in t_{\mathcal{D}^{\prime}}$, then $a=d=0, b=\sum_{k \in I_{1}} b_{k}\left(\sum_{g \in C}\left(\chi / \chi^{\sigma}\right)(g) g\right)+\sum_{k \in I_{2}} b_{k}\left(\sum_{g \in C}\left(\chi / \chi^{\sigma}\right)(g) g \sigma^{\prime}\right)$ and $c=\sum_{k \in I_{1}} b_{k}\left(\sum_{g \in G \backslash C}\left(\chi / \chi^{\sigma}\right)\left(\sigma^{\prime} g\right) g\right)+\sum_{k \in I_{2}} b_{k}\left(\sum_{g \in C}\left(\chi^{\sigma} / \chi\right)(g) g\right)$. Now, suppose $b \neq 0$. It follows that $\left(b_{1}, \cdots, b_{n}\right) \neq(0, \cdots, 0)$. As $b \in \operatorname{Hom}\left(G_{H}, \overline{\mathbb{Q}}_{p}\right)$, then $b\left(x_{l}\right)=0$ for $l=1, \cdots, n$. Thus, $\sum_{j \in I_{1}} b_{j} \sum_{g \in C}\left(\chi / \chi^{\sigma}\right)(g) \log _{p}\left(i_{p} \circ i_{j}^{\prime}\left(g^{-1}\left(x_{l}\right)\right)\right)+$ 
$\sum_{j \in I_{2}} b_{j} \sum_{g \in C}\left(\chi / \chi^{\sigma}\right)(g) \log _{p}\left(i_{p} \circ i_{j}^{\prime}\left(\sigma^{\prime} g^{-1}\left(x_{l}\right)\right)\right)=0$ for $l=1, \cdots, n$. Hence, the $n \times n$ matrix $\left(\sum_{g \in C}\left(\chi / \chi^{\sigma}\right)(g) \log _{p}\left(i_{p} \circ i_{j}^{\prime}\left(\left(g^{-1}\left(x_{l}\right)\right)_{j}\right)\right)\right)_{1 \leq j \leq n, 1 \leq l \leq n}$ is not invertible, where $\left(g^{-1}\left(x_{l}\right)\right)_{j}=g^{-1}\left(x_{l}\right)$ if $j \in I_{1}$ and $\left(g^{-1}\left(x_{l}\right)\right)_{j}=\sigma^{\prime} g^{-1}\left(x_{l}\right)$ if $j \in I_{2}$. Thus, its determinant is zero that is, $\operatorname{det}\left(\sum_{g \in C}\left(\chi / \chi^{\sigma}\right)(g) \log _{p}\left(i_{p} \circ i_{j}^{\prime}\left(\left(g^{-1}\left(x_{l}\right)\right)_{j}\right)\right)\right)_{1 \leq j \leq n, 1 \leq l \leq n}=0$.

We will view all the units above as elements of the Galois closure $H^{\prime}$ of $H$ over $\mathbb{Q}$. Let $M$ be the $\mathbb{Z}$-submodule of $\mathcal{O}_{H^{\prime}}^{\times}$generated by elements of the set $\left\{i_{j}^{\prime}\left(g^{-1}\left(x_{l}\right)\right)\right\}_{j \in I_{1}, g \in C, 1 \leq l \leq n} \cup\left\{i_{j}^{\prime}\left(\sigma^{\prime} g^{-1}\left(x_{l}\right)\right)\right\}_{j \in I_{2}, g \in C, 1 \leq l \leq n}$ and let $\left\{u_{1}, \cdots, u_{m}\right\}$ be the subset of $\left\{i_{j}^{\prime}\left(g^{-1}\left(x_{l}\right)\right)\right\}_{j \in I_{1}, g \in C, 1 \leq l \leq n} \cup\left\{i_{j}^{\prime}\left(\sigma^{\prime} g^{-1}\left(x_{l}\right)\right)\right\}_{j \in I_{2}, g \in C, 1 \leq l \leq n}$ such that it forms a basis of $M \otimes_{\mathbb{Z}} \mathbb{Q}$. So, we can see the determinant as a polynomial in $\log _{p}\left(i_{p}\left(u_{1}\right)\right), \cdots, \log _{p}\left(i_{p}\left(u_{m}\right)\right)$ with coefficients in $\overline{\mathbb{Q}}$. Call the polynomial $P$ which will be a polynomial in $m$ variables. Recall that the $p$-adic Schanuel conjecture ( 8 , Conjecture 3.10]) states that if $\alpha_{1}, \cdots, \alpha_{n} \in \overline{\mathbb{Q}}$ are such that $\log _{p}\left(\alpha_{1}\right), \cdots, \log _{p}\left(\alpha_{n}\right)$ are linearly independent over $\mathbb{Q}$, then the field $\mathbb{Q}\left(\log _{p}\left(\alpha_{1}\right), \cdots, \log _{p}\left(\alpha_{n}\right)\right)$ has transcendence degree $n$ over $\mathbb{Q}$. Now assume that the $p$-adic Schanuel conjecture. Then it follows that $\mathbb{Q}\left(\log _{p}\left(i_{p}\left(u_{1}\right)\right), \cdots, \log _{p}\left(i_{p}\left(u_{m}\right)\right)\right)$ has transcendence degree $m$ over $\mathbb{Q}$. But $P$ is a polynomial over $\overline{\mathbb{Q}}$ which is satisfied by $\log _{p}\left(i_{p}\left(u_{1}\right)\right), \cdots, \log _{p}\left(i_{p}\left(u_{m}\right)\right)$. Hence, all the coefficients of $P$ are zero. Observe that, on the complex side, we have $\operatorname{det}\left(\sum_{g \in C}\left(\chi / \chi^{\sigma}\right)(g) \log \left|i_{j}^{\prime}\left(\left(g^{-1}\left(x_{l}\right)\right)_{j}\right)\right|\right)=P\left(\log \left(\left|u_{1}\right|\right), \cdots, \log \left(\left|u_{m}\right|\right)\right)$. But as all the coefficients of $P$ are zero, we see that the determinant $\operatorname{det}\left(\sum_{g \in C}\left(\chi / \chi^{\sigma}\right)(g) \log \left|i_{j}^{\prime}\left(\left(g^{-1}\left(x_{l}\right)\right)_{j}\right)\right|\right)_{1 \leq j \leq n, g \in C, 1 \leq l \leq n}=0$.

Note that, the set of left coset representatives of $G /\left\{1, \tau_{j}\right\}$ can be given by $C$ for $j \in I_{1}$ and by $C \sigma^{\prime}$ for $j \in I_{2}$. We can identify $\mathbb{C}^{\frac{n|G|}{2}}$ with $G$-representation $\oplus_{j=1}^{n} \operatorname{Ind}_{\left\{1, \tau_{j}\right\}}^{G} 1$. Indeed, we can label the standard basis of $\mathbb{C}^{\frac{n|G|}{2}}$ by $\left\{i_{j}^{\prime} \circ g\right\}_{j \in I_{1}, g \in C} \cup$ $\left\{i_{j}^{\prime} \circ g \sigma^{\prime}\right\}_{j \in I_{2}, g \in C}$ and give the action of $G$ by $g^{\prime} \cdot e_{i_{j}^{\prime} \circ g}=e_{i_{j}^{\prime} \circ\left[g^{\prime} g\right]}$ where, if $j \in I_{1}$, then $\left[g^{\prime} g\right]$ is the element in $C$ which represents the left coset of $g^{\prime} g$ in $G /\left\{1, \tau_{j}\right\}$ and if $j \in I_{2}$, then $\left[g^{\prime} g\right]$ is the element in $C \sigma^{\prime}$ which represents the left coset of $g^{\prime} g$ in $G /\left\{1, \tau_{j}\right\}$. Let $\phi: \mathcal{O}_{H}^{\times} \otimes_{\mathbb{Z}} \overline{\mathbb{Q}} \rightarrow \mathbb{C}^{\frac{n|G|}{2}}$ be the linear map given by $u \otimes$ $1 \mapsto\left(\left(\log \left|i_{j}^{\prime}\left(g^{-1}(u)\right)\right|\right)_{j \in I_{1}, g \in C},\left(\log \left|i_{j}^{\prime}\left(\sigma^{\prime} g^{-1}(u)\right)\right|\right)_{j \in I_{2}, g \in C}\right)$. By Minkowski's proof of Dirichlet's unit theorem, we know that $\phi$ is an injective map which is $G$-equivariant and the $\mathbb{C}$-vector space generated by $\operatorname{Im}(\phi)$ is of dimension $\frac{n|G|}{2}-1$ and is isomorphic to $\left(\oplus_{j=1}^{n} \operatorname{Ind}_{\left\{1, \tau_{j}\right\}}^{G} 1\right) \backslash 1$ as a $G$-representation.

Thus, we see that $\phi\left(\left(\sum_{g \in C}\left(\chi / \chi^{\sigma}\right)(g) g^{-1}\right) x_{1}\right), \cdots, \phi\left(\left(\sum_{g \in C}\left(\chi / \chi^{\sigma}\right)(g) g^{-1}\right) x_{n}\right)$ are linearly independent vectors and $C$ acts on them by $\chi / \chi^{\sigma}$. So, it follows that, for all $j \in I_{1} \phi\left(\left(\sum_{g \in C}\left(\chi / \chi^{\sigma}\right)(g) g^{-1}\right) x_{k}\right)_{i_{j}^{\prime} \circ g^{\prime}}=\left(\chi^{\sigma} / \chi\right)\left(g^{\prime}\right)\left(\phi\left(\left(\sum_{g \in C}\left(\chi / \chi^{\sigma}\right)(g) g^{-1}\right) x_{k}\right)_{i_{j}^{\prime} \circ 1}\right)$ for $1 \leq k \leq n$ and $g^{\prime} \in C$. Similarly, for all $j \in I_{2}, \phi\left(\left(\sum_{g \in C}\left(\chi / \chi^{\sigma}\right)(g) g^{-1}\right) x_{k}\right)_{i_{j}^{\prime} \circ g^{\prime} \sigma^{\prime}}$ 
$=\left(\chi^{\sigma} / \chi\right)\left(g^{\prime}\right)\left(\phi\left(\left(\sum_{g \in C}\left(\chi / \chi^{\sigma}\right)(g) g^{-1}\right) x_{k}\right)_{i_{j}^{\prime} \circ \sigma^{\prime}}\right)$ for $1 \leq k \leq n$ and $g^{\prime} \in C$. So the $n$ tuples $\left(\left(\sum_{g \in C}\left(\chi / \chi^{\sigma}\right)(g) \log \left|i_{j}^{\prime}\left(g^{-1}\left(x_{1}\right)\right)\right|\right)_{j \in I_{1}},\left(\sum_{g \in C}\left(\chi / \chi^{\sigma}\right)(g) \log \left|i_{j}^{\prime}\left(\sigma^{\prime} g^{-1}\left(x_{1}\right)\right)\right|\right)_{j \in I_{2}}\right)$, $\cdots,\left(\left(\sum_{g \in C}\left(\chi / \chi^{\sigma}\right)(g) \log \left|i_{j}^{\prime}\left(g^{-1}\left(x_{n}\right)\right)\right|\right)_{j \in I_{1}},\left(\sum_{g \in C}\left(\chi / \chi^{\sigma}\right)(g) \log \left|i_{j}^{\prime}\left(\sigma^{\prime} g^{-1}\left(x_{n}\right)\right)\right|\right)_{j \in I_{2}}\right)$ are linearly independent (these vectors are obtained by taking $\left(i_{j}^{\prime} \circ 1\right)$-th co-ordinates for $j \in I_{1}$ and $\left(i_{j}^{\prime} \circ \sigma^{\prime}\right)$-th co-ordinates for $j \in I_{2}$ of the $n$ linearly independent vectors given above). Therefore, using the notation introduced above, we get that the matrix $\left(\sum_{g \in C}\left(\chi / \chi^{\sigma}\right)(g) \log \left|i_{j}^{\prime}\left(\left(g^{-1}\left(x_{l}\right)\right)_{j}\right)\right|\right)_{1 \leq j \leq n, 1 \leq l \leq n}$ is invertible and $\operatorname{det}\left(\sum_{g \in C}\left(\chi / \chi^{\sigma}\right)(g) \log \left|i_{j}^{\prime}\left(\left(g^{-1}\left(x_{l}\right)\right)_{j}\right)\right|\right)_{1 \leq j \leq n, 1 \leq l \leq n} \neq 0$. But we already concluded above, after assuming $\left(b_{1}, \cdots, b_{n}\right) \neq(0, \cdots, 0)$ and the $p$-adic Schanuel conjecture, that $\operatorname{det}\left(\sum_{g \in C}\left(\chi / \chi^{\sigma}\right)(g) \log \left|i_{j}^{\prime}\left(\left(g^{-1}\left(x_{l}\right)\right)_{j}\right)\right|_{1 \leq j \leq n, 1 \leq l \leq n}=0\right.$. Thus, we get a contradiction to our assumption that $\left(b_{1}, \cdots, b_{n}\right) \neq(0, \cdots, 0)$ after assuming the $p$-adic Schanuel conjecture. Hence, after assuming the $p$-adic Schanuel conjecture, we see that $\operatorname{dim} t_{\mathcal{D}^{\prime}}=0$.

If the $p$-adic Schanuel conjecture is true, then by [17, Theorem 6.4], the Leopoldt conjecture is also true. Hence, Proposition 1 implies that $\operatorname{dim} t_{\mathcal{D}_{0}}=1$ and $\operatorname{dim} t_{\mathcal{D}}=$ $n+1$.

Suppose $\rho=\operatorname{Ind}_{G_{K}}^{G_{F}} \chi, G$ is a non-abelian dihedral group, $K$ has exactly $2(n-s)$ real embeddings and all primes of $F$ above $p$ are split in $K$. So, we see (after relabeling, if necessary) $\tau_{1}, \cdots, \tau_{s} \in G \backslash C$ and $\tau_{s+1}=\cdots=\tau_{n}=\tau$. Thus, $\operatorname{Ind}_{G_{K}}^{G_{F}}\left(\chi / \chi^{\sigma}\right)$ appears in $\mathcal{O}_{H}^{\times} \otimes_{\mathbb{Z}} \overline{\mathbb{Q}}$ with multiplicity $s$ and $\epsilon_{K}$ appears in it with multiplicity $n-s$. Hence, the subspace $V_{\chi / \chi^{\sigma}}$ of $\mathcal{O}_{H}^{\times} \otimes_{\mathbb{Z}} \overline{\mathbb{Q}}$ on which $C$ acts by $\chi / \chi^{\sigma}$ has dimension $s$. Let $x_{1}, \cdots, x_{s} \in \mathcal{O}_{H}^{\times}$be such that a basis of $V_{\chi / \chi^{\sigma}}$ is given by $\left\{\left(\sum_{g \in C}\left(\chi / \chi^{\sigma}\right)(g) g^{-1}\right)\left(x_{1} \otimes 1\right), \cdots,\left(\sum_{g \in C}\left(\chi / \chi^{\sigma}\right)(g) g^{-1}\right)\left(x_{s} \otimes 1\right)\right\}$ (it is clear that we can find such units). Therefore, if the Leopoldt conjecture is true for $H$, then $\operatorname{Ind}_{G_{K}}^{G_{F}}\left(\chi / \chi^{\sigma}\right)$ appears in $\operatorname{Hom}\left(G_{H}, \overline{\mathbb{Q}}_{p}\right)$ with multiplicity $2 n-s$ and $\epsilon_{K}$ appears in it with multiplicity $s$.

Fix the basis $\left(v_{1}, v_{2}\right)$ as in the previous section. As all primes of $F$ above $p$ are split in $K$, from the formulas we found earlier, it follows that if $\left(\begin{array}{ll}a & b \\ c & d\end{array}\right) \in t_{\mathcal{D}^{\prime}}$, then

$$
\left(\begin{array}{ll}
a & b \\
c & d
\end{array}\right)=\sum_{k \in I_{1}} \sum_{g \in G}\left(\rho(g)\left(\begin{array}{cc}
0 & b_{k} \\
0 & 0
\end{array}\right) \rho(g)^{-1}\right) g+\sum_{k \in I_{2}} \sum_{g \in G}\left(\rho(g)\left(\begin{array}{cc}
0 & 0 \\
b_{k} & 0
\end{array}\right) \rho(g)^{-1}\right) g
$$

Hence, we easily see that $a=d=0, b=\sum_{k \in I_{1}} b_{k}\left(\sum_{g \in C}\left(\chi / \chi^{\sigma}\right)(g) g\right)+\sum_{k \in I_{2}} b_{k}\left(\sum_{g \in C}\left(\chi / \chi^{\sigma}\right)(g) g \sigma^{\prime}\right)$ and $c=\sum_{k \in I_{1}} b_{k}\left(\sum_{g \in G \backslash C}\left(\chi / \chi^{\sigma}\right)\left(\sigma^{\prime} g\right) g\right)+\sum_{k \in I_{2}} b_{k}\left(\sum_{g \in C}\left(\chi^{\sigma} / \chi\right)(g) g\right)$. Recall that, $b$ and $c$ together generate $\operatorname{Ind}_{G_{K}}^{G_{F}}\left(\chi / \chi^{\sigma}\right)$ which appears in $\operatorname{Hom}\left(G_{H}, \overline{\mathbb{Q}}_{p}\right)$ with multiplicity $2 n-s$. Each of these $(2 n-s)$ copies is obtained by replacing $\left(\begin{array}{cc}0 & b_{k} \\ 0 & 0\end{array}\right)$ 
with a suitable matrix $\left(\begin{array}{cc}0 & b_{k}^{\prime} \\ c_{k} & 0\end{array}\right)$ and by replacing $\left(\begin{array}{cc}0 & 0 \\ b_{k} & 0\end{array}\right)$ with a suitable matrix $\left(\begin{array}{cc}0 & c_{k} \\ b_{k}^{\prime} & 0\end{array}\right)$ in the equation above and taking the space generated by the resulting $b$ and $c$. Thus, the copies of $\operatorname{Ind}_{G_{K}}^{G_{F}}\left(\chi / \chi^{\sigma}\right)$ obtained from $t_{\mathcal{D}^{\prime}}$ are obtained from these $2 n-s$ copies by putting the $n$ extra conditions that $c_{1}=\cdots=c_{n}=0$. Hence, $\operatorname{dim} t_{\mathcal{D}^{\prime}} \geq(2 n-s)-n=n-s$.

Now, suppose $\operatorname{dim} t_{\mathcal{D}^{\prime}}>n-s$ i.e. the dimension of the vector space generated by $\left(b_{1}, \cdots, b_{n}\right)$ is greater than $n-s$. As $b \in \operatorname{Hom}\left(G_{H}, \overline{\mathbb{Q}}_{p}\right)$, then $b\left(x_{l}\right)=0$ for $l=1, \cdots, s$. Thus, we get $\sum_{j \in I_{1}} b_{j} \sum_{g \in C}\left(\chi / \chi^{\sigma}\right)(g) \log _{p}\left(i_{p} \circ i_{j}^{\prime}\left(g^{-1}\left(x_{l}\right)\right)\right)$ $+\sum_{j \in I_{2}} b_{j} \sum_{g \in C}\left(\chi / \chi^{\sigma}\right)(g) \log _{p}\left(i_{p} \circ i_{j}^{\prime}\left(\sigma^{\prime} g^{-1}\left(x_{l}\right)\right)\right)=0$ for $l=1, \cdots, s$. Hence, the rank of the $s \times n$ matrix $\left(\sum_{g \in C}\left(\chi / \chi^{\sigma}\right)(g) \log _{p}\left(i_{p} \circ i_{j}^{\prime}\left(\left(g^{-1}\left(x_{l}\right)\right)_{j}\right)\right)\right)_{1 \leq j \leq n, 1 \leq l \leq s}$ is less than $s$, where $\left(g^{-1}\left(x_{l}\right)\right)_{j}=g^{-1}\left(x_{l}\right)$ if $j \in I_{1}$ and $\left(g^{-1}\left(x_{l}\right)\right)_{j}=\sigma^{\prime} g^{-1}\left(x_{l}\right)$ if $j \in I_{2}$. As a consequence, the determinant of every $s \times s$ minor of this matrix is 0 . In particular, $\operatorname{det}\left(\sum_{g \in C}\left(\chi / \chi^{\sigma}\right)(g) \log _{p}\left(i_{p} \circ i_{j}^{\prime}\left(\left(g^{-1}\left(x_{l}\right)\right)_{j}\right)\right)\right)_{1 \leq j \leq s, 1 \leq l \leq s}=0$. Using the same logic as used in the CM case above, we see that, after assuming the $p$-adic Schanuel conjecture, $\operatorname{det}\left(\sum_{g \in C}\left(\chi / \chi^{\sigma}\right)(g) \log \left|i_{j}^{\prime}\left(\left(g^{-1}\left(x_{l}\right)\right)_{j}\right)\right|\right)_{1 \leq j \leq s, 1 \leq l \leq s}=0$.

As in the previous subcase (CM case) above, we can label the standard basis of $\mathbb{C}^{\frac{n|G|}{2}}$ by $\left\{i_{j}^{\prime} \circ g_{j}\right\}_{1 \leq j \leq n, g_{j} \in G /\left\{1, \tau_{j}\right\}}$ and give the action of $G$ by $g^{\prime} \cdot e_{i_{j}^{\prime} \circ g_{j}}=e_{i_{j}^{\prime} \circ\left[g^{\prime} g_{j}\right]}$ where $\left[g^{\prime} g_{j}\right]$ is the element in $G /\left\{1, \tau_{j}\right\}$ which represents the left coset of $g^{\prime} g_{j}$ in $G /\left\{1, \tau_{j}\right\}$. This identifies $\mathbb{C}^{\frac{n|G|}{2}}$ with $\oplus_{j=1}^{n} \operatorname{Ind}_{\left\{1, \tau_{j}\right\}}^{G} 1$ in a way that makes the linear $\operatorname{map} \phi: \mathcal{O}_{H}^{\times} \otimes_{\mathbb{Z}} \overline{\mathbb{Q}} \rightarrow \mathbb{C}^{\frac{n|G|}{2}}$ given by $u \otimes 1 \mapsto\left(\log \left|i_{j}^{\prime}\left(g_{j}^{-1}(u)\right)\right|\right)_{1 \leq j \leq n, g_{j} \in G /\left\{1, \tau_{j}\right\}} G$ equivariant. Note that, the set of left coset representatives of $G /\left\{1, \tau_{j}\right\}$ can be given by $C$ for all $1 \leq j \leq s$ such that $j \in I_{1}$ and by $C \sigma^{\prime}$ for all $1 \leq j \leq s$ such that $j \in I_{2}$, so we will use it for coset representatives for $1 \leq j \leq s$ in the map above. From Minkowski's proof of Dirichlet's unit theorem, we know that this map is injective and the $\mathbb{C}$-vector space generated by $\operatorname{Im}(\phi)$ has dimension $\frac{n|G|}{2}-1$ and is isomorphic to $\left(\oplus_{j=1}^{n} \operatorname{Ind}_{\left\{1, \tau_{j}\right\}}^{G} 1\right) \backslash 1$ as a $G$-representation.

Thus, we see that $\phi\left(\left(\sum_{g \in C}\left(\chi / \chi^{\sigma}\right)(g) g^{-1}\right) x_{1}\right), \cdots, \phi\left(\left(\sum_{g \in C}\left(\chi / \chi^{\sigma}\right)(g) g^{-1}\right) x_{s}\right)$ are linearly independent vectors and $C$ acts on them by $\chi^{\sigma} / \chi$. Thus, $\phi\left(\left(\sum_{g \in C}\left(\chi / \chi^{\sigma}\right)(g) g^{-1}\right) x_{k}\right)_{i_{j}^{\prime} \circ g^{\prime}}$ $=\left(\chi^{\sigma} / \chi\right)\left(g^{\prime}\right)\left(\phi\left(\left(\sum_{g \in C}\left(\chi / \chi^{\sigma}\right)(g) g^{-1}\right) x_{j}\right)_{i_{k}^{\prime} \circ 1}\right)$ for all $j \in I_{1}$ with $1 \leq j \leq s, 1 \leq k \leq s$ and $g^{\prime} \in C$ and $\phi\left(\left(\sum_{g \in C}\left(\chi / \chi^{\sigma}\right)(g) g^{-1}\right) x_{k}\right)_{i_{j}^{\prime} \circ g^{\prime} \sigma^{\prime}}=\left(\chi^{\sigma} / \chi\right)\left(g^{\prime}\right)\left(\phi\left(\left(\sum_{g \in C}\left(\chi / \chi^{\sigma}\right)(g) g^{-1}\right) x_{j}\right)_{i_{k}^{\prime} \circ \sigma^{\prime}}\right)$ for all $j \in I_{2}$ with $1 \leq j \leq s, 1 \leq k \leq s$ and $g^{\prime} \in C$. Note that, the subrepresentation of $\mathbb{C}^{\frac{n|G|}{2}}$ given by $\oplus_{j=s+1}^{n} \operatorname{Ind}_{\left\{1, \tau_{j}\right\}}^{G} 1$, which is generated co-ordinates corresponding to $i_{j}^{\prime} \circ g_{j^{\prime}}$ with $s+1 \leq j \leq n$ and $g_{j^{\prime}} \in G /\left\{1, \tau_{j}\right\}$, does not contain $\operatorname{Ind}_{G_{K}}^{G_{F}}\left(\chi / \chi^{\sigma}\right)$. Thus, it does not contain any non-zero vector on which $C$ acts like 
$\chi / \chi^{\sigma}$. So, $\phi\left(\left(\sum_{g \in C}\left(\chi / \chi^{\sigma}\right)(g) g^{-1}\right) x_{k}\right)_{i_{j}^{\prime} \circ g_{j^{\prime}}}=0$ for all $1 \leq k \leq s, s+1 \leq j \leq n$ and $g_{j^{\prime}} \in G /\left\{1, \tau_{j}\right\}$.

So, we see that the $s$-tuples $\left(\sum_{g \in C}\left(\chi / \chi^{\sigma}\right)(g) \log \left|i_{j}^{\prime}\left(\left(g^{-1}\left(x_{1}\right)\right)_{j}\right)\right|\right)_{1 \leq j \leq s}, \cdots$, $\left(\sum_{g \in C}\left(\chi / \chi^{\sigma}\right)(g) \log \left|i_{j}^{\prime}\left(\left(g^{-1}\left(x_{s}\right)\right)_{j}\right)\right|\right)_{1 \leq j \leq s}$ are linearly independent (these vectors are obtained by taking $\left(i_{j}^{\prime} \circ 1\right)$-th co-ordinates for $j \in I_{1}$ with $1 \leq j \leq s$ and by taking $\left(i_{j}^{\prime} \circ \sigma^{\prime}\right)$-th co-ordinates for $j \in I_{2}$ with $1 \leq j \leq s$ of the $s$ linearly independent vectors given above). Therefore, the matrix $\left(\sum_{g \in C}\left(\chi / \chi^{\sigma}\right)(g) \log \left|i_{j}^{\prime}\left(\left(g^{-1}\left(x_{l}\right)\right)_{j}\right)\right|\right)_{1 \leq j \leq s, 1 \leq l \leq s}$ is invertible and $\operatorname{det}\left(\sum_{g \in C}\left(\chi / \chi^{\sigma}\right)(g) \log \left|i_{j}^{\prime}\left(\left(g^{-1}\left(x_{l}\right)\right)_{j}\right)\right|\right)_{1 \leq j \leq s, 1 \leq l \leq s} \neq 0$. But we already concluded above, that $\operatorname{det}\left(\sum_{g \in C}\left(\chi / \chi^{\sigma}\right)(g) \log \left|i_{j}^{\prime}\left(\left(g^{-1}\left(x_{l}\right)\right)_{j}\right)\right|\right)_{1 \leq j \leq s, 1 \leq l \leq s}=0$. Thus, we get a contradiction to our assumption that $\operatorname{dim} t_{\mathcal{D}^{\prime}}>n-s$, after assuming the $p$-adic Schanuel conjecture. Hence, after assuming the $p$-adic Schanuel conjecture, we see that $\operatorname{dim} t_{\mathcal{D}^{\prime}}=n-s$.

As all primes of $F$ above $p$ are split in $K$, from $\left[5\right.$, , it follows that if $\left(\begin{array}{ll}a & b \\ c & d\end{array}\right) \in t_{\mathcal{D}}$, then

$$
\left(\begin{array}{ll}
a & b \\
c & d
\end{array}\right)=\sum_{k \in I_{1}} \sum_{g \in G}\left(\rho(g)\left(\begin{array}{cc}
a_{k} & b_{k} \\
0 & d_{k}
\end{array}\right) \rho(g)^{-1}\right) g+\sum_{k \in I_{2}} \sum_{g \in G}\left(\rho(g)\left(\begin{array}{cc}
d_{k} & 0 \\
b_{k} & a_{k}
\end{array}\right) \rho(g)^{-1}\right) g
$$

Recall that, $a+d$ generates the trivial representation 1, while $a-d$ generates $\epsilon_{K}$ and their expressions do not involve $b_{k}$ 's. So, if $a_{k}$ 's, $b_{k}$ 's and $d_{k}$ 's are giving an element of $t_{\mathcal{D}}$ after their substitution in the formula above, we should get an element of $t_{\mathcal{D}}$ after making all the $b_{k}$ 's 0 .

If the $p$-adic Schanuel conjecture is true, then by [17, Theorem 6.4], the Leopoldt conjecture is also true. So, $\epsilon_{K}$ appears in $\operatorname{Hom}\left(G_{H}, \overline{\mathbb{Q}}_{p}\right)$ with multiplicity $s$ and 1 appears with multiplicity one. So, we see that $a_{1}+d_{1}=\cdots=a_{n}+d_{n}$ and the vector space generated by $n$-tuples $\left(\left(a_{j}-d_{j}\right)_{j \in I_{1}},\left(d_{j}-a_{j}\right)_{j \in I_{2}}\right)$ coming from all possible $a_{k}$ 's and $d_{k}$ 's should have dimension $s$. Hence, the dimension of the space generated by the $2 n$-tuples $\left(a_{1}, \cdots, a_{n}, d_{1}, \cdots, d_{n}\right)$ which give rise to an element of $t_{\mathcal{D}}$ when substituted in the equation above with $b_{1}=\cdots=b_{n}=0$ has dimension $s+1$.

Thus, combining all the observations above, we see that $\operatorname{dim} t_{\mathcal{D}}=\operatorname{dim} t_{\mathcal{D}^{\prime}}+s+1=$ $(n-s)+s+1=n+1$. It follows, from Proposition 1, that $\operatorname{dim} t_{\mathcal{D}_{0}} \leq n-s+1$ and from the computation of $\operatorname{dim} t_{\mathcal{D}^{\prime}}$ above, we see that $\operatorname{dim} t_{\mathcal{D}_{0}} \geq n-s$. This concludes the proof of Theorem 1 for the third case.

\section{Ordinary locus of the Eigenvariety AND its Properties}

As in the introduction $(\S 1)$, let $\mathcal{E}$ be the $p$-adic eigenvariety parameterizing cuspidal Hilbert modular eigenforms of tame level $N$ defined over $F$. It is constructed 
using the Hecke operators $U_{\mathfrak{p}_{i}}$ for $\mathfrak{p}_{i} \mid p$ and $T(\mathfrak{q})$ for primes $\mathfrak{q} \nmid N p$. It is reduced and there is a locally finite morphism $\kappa: \mathcal{E} \rightarrow \mathcal{W}$, known as the weight map such that, for $x \in \mathcal{E}$, the local ring of $\mathcal{E}$ at $x$ is a torsion-free module of finite type over the local ring of $\mathcal{W}$ at $\kappa(x)$ (see [1] for more details about the construction). Note that, by [7, Lemma 5.8], $\mathcal{E}$ is equi-dimensional of dimension $n+1$ (see [1, Theorem 5.1]). By construction, we have analytic functions $U\left(\mathfrak{p}_{i}\right) \in \mathcal{O}(\mathcal{E})^{\times}$for $\mathfrak{p}_{i} \mid p$ and $T(\mathfrak{q}) \in \mathcal{O}(\mathcal{E})$ for $\mathfrak{q} \nmid N p$. By [1, Theorem 5.1], there exists a continuous pseudo-character $G_{F} \rightarrow \mathcal{O}(\mathcal{E})$ which sends Frob $_{\mathfrak{q}}$ to $T(\mathfrak{q})$ for all primes $\mathfrak{q} \nmid N p$. This pseudo-character is obtained by the techniques illustrated in [3] using other properties of the eigenvariety.

If $p=\prod_{i=1}^{r} \mathfrak{p}_{i}{ }_{i}$ is the prime decomposition of $p$ in $\mathcal{O}_{F}$, then let $U(p)=\prod_{i=1}^{r} U\left(\mathfrak{p}_{i}\right)^{e_{i}}$ $\in \mathcal{O}(\mathcal{E})^{\times}$. The locus where $|U(p)|_{p}=1$ is both open and closed in $\mathcal{E}$, and we call it the ordinary locus of the eigenvariety. It is closely related to nearly ordinary Hida families. The system of eigenvalues of a refinement $f_{\left(\gamma_{i}\right)}$ of $f$ (as in the introduction) corresponds to a point $x \in \mathcal{E}\left(\bar{Q}_{p}\right)$ such that $\kappa(x)$ has finite order. As $U\left(\mathfrak{p}_{i}\right)(x)=\gamma_{i}$ is a $p$-adic unit for every $1 \leq i \leq r, x$ lies on the ordinary locus of $\mathcal{E}$. Let $\mathcal{T}$ be the local ring of $\mathcal{E}$ at $x$ and let $\mathfrak{m}$ be its maximal ideal. So, the Krull dimension of $\mathcal{T}$ is $n+1$.

The construction of the eigenvariety $\mathcal{E}$ in the parallel weight case is equivalent to the parallel weight eigenvariety $\mathcal{C}$ constructed by Kisin and Lai in [16]. Thus, $\mathcal{C}^{\text {cusp }}$, the cuspidal part of $\mathcal{C}$, can be seen as a closed subvariety of $\mathcal{E}$. This gives a map $\alpha: \mathcal{O}(\mathcal{E}) \rightarrow \mathcal{O}\left(\mathcal{C}^{\text {cusp }}\right)$ which induces a surjective map $\mathcal{O}_{\mathcal{E}, x} \rightarrow \mathcal{O}_{\mathcal{C}^{\text {cusp }, \alpha(x)}}$ for all $x \in \mathcal{E}$, where $\mathcal{O}_{\mathcal{E}, x}$ is the local ring of $\mathcal{E}$ at $x$ and $\mathcal{O}_{\mathcal{C}^{\text {cusp }}, \alpha(x)}$ is the local ring of $\mathcal{C}^{\text {cusp }}$ at $\alpha(x)$. Indeed, the map on local rings is surjective as both of them are generated by the Hecke operators $T(\mathfrak{q})$ for primes $\mathfrak{q} \nmid N p$ and $U\left(\mathfrak{p}_{i}\right)$ for primes $\mathfrak{p}_{i} \mid p$ and the map $\alpha$ is the restriction map. All the properties of $\mathcal{E}$ listed above also hold for $\mathcal{C}^{\text {cusp }}$ (after changing the weight space suitably) and $x$ lies in $\mathcal{C}^{\text {cusp }}$. In particular, we get a continuous pseudo-character $G_{F} \rightarrow \mathcal{O}\left(\mathcal{C}^{\text {cusp }}\right)$ which sends Frob $\mathfrak{q}_{\text {to }} T(\mathfrak{q})$ for all primes $\mathfrak{q} \nmid N p$. We can define the ordinary locus of $\mathcal{C}^{\text {cusp }}$ in the same way. It will be an open and closed subspace of $\mathcal{C}^{\text {cusp }}$ and moreover, $x$ lies in the ordinary locus of $\mathcal{C}$. Let $\mathcal{T}_{0}$ be the local ring of $\mathcal{C}^{\text {cusp }}$ at $x$ with maximal ideal $\mathfrak{m}_{0}$. Thus, we have a surjective map $\mathcal{T} \rightarrow \mathcal{T}_{0}$. The Krull dimension of $\mathcal{T}_{0}$ is 1 .

Proposition 4. (1) There exists a continuous representation $\rho_{\mathcal{T}}: G_{F} \rightarrow G L_{2}(\mathcal{T})$, such that $\operatorname{Tr}\left(\rho_{T}\left(\right.\right.$ Frob $\left.\left._{\mathfrak{q}}\right)\right)=T(\mathfrak{q})$ for all primes $\mathfrak{q} \nmid N p$. The reduction of $\rho_{\mathcal{T}}$ modulo $\mathfrak{m}$ is $\rho$. As $f$ is regular at $p, \rho_{\mathcal{T}}$ is nearly ordinary at every $\mathfrak{p}_{i} \mid p$ in the sense that: $\left(\rho_{\mathcal{T}}\right) \mid G_{F_{\mathfrak{p}_{i}}} \simeq\left(\begin{array}{cc}\left(\psi_{\mathcal{T}}^{\prime}\right)_{i} & * \\ 0 & \left(\psi_{\mathcal{T}}^{\prime \prime}\right)_{i}\end{array}\right)$ and there exists a lift of Frob $\mathfrak{p}_{i}$ 
in $G_{F_{\mathfrak{p}_{i}}}$ which gets mapped to $U\left(\mathfrak{p}_{i}\right)$ under the character $\left(\psi_{\mathcal{T}}^{\prime \prime}\right)_{i}: G_{F_{\mathfrak{p}_{i}}} \rightarrow \mathcal{T}^{\times}$ for all $1 \leq i \leq r$.

(2) The continuous representation $\rho_{\mathcal{T}_{0}}: G_{F} \rightarrow G L_{2}\left(\mathcal{T}_{0}\right)$, obtained by composing $\rho_{\mathcal{T}}$ with the surjective map $\mathcal{T} \rightarrow \mathcal{T}_{0}$, is ordinary at every $\mathfrak{p}_{i} \mid p$ in the sense that: $\left(\rho_{\mathcal{T}_{0}}\right) \mid G_{F_{\mathfrak{p}_{i}}} \simeq\left(\begin{array}{cc}\left(\psi_{\mathcal{T}_{0}}^{\prime}\right)_{i} & * \\ 0 & \left(\psi_{\mathcal{T}_{0}}^{\prime \prime}\right)_{i}\end{array}\right)$ and $\left(\psi_{\mathcal{T}_{0}}^{\prime \prime}\right)_{i}: G_{F_{\mathfrak{p}_{i}}} \rightarrow \mathcal{T}_{0} \times$ is the unramified character sending Frob $\mathfrak{p}_{i}$ to $U\left(\mathfrak{p}_{i}\right)$ for all $1 \leq i \leq r$.

Proof. Same as the proof of Proposition 5.1 of [4]. We get the representation $\rho_{\mathcal{T}}$ from the pseudo-character $G_{F, N p} \rightarrow \mathcal{T}$ obtained by composing the pseudo-character $G_{F, N p} \rightarrow \mathcal{O}(\mathcal{E})$ with the map $\mathcal{O}(\mathcal{E})$. To prove that $\rho_{\mathcal{T}}$ is nearly ordinary at every $\mathfrak{p}_{i}$, we can use the exact same argument that is given in the proof of Proposition 5.1 of [4] for every prime $\mathfrak{p}_{i}$ by replacing [23, Theorem 2.2.2] with [14, Proposition 2.3] in their argument. Note that we can use their argument for all primes $\mathfrak{p}_{i}$ because we have assumed that $f$ is regular at every $\mathfrak{p}_{i}$. To prove part (2), we can again use the exact same argument of [4] mentioned above and we don't even need to use [14, Proposition 2.3] instead of [23, Theorem 2.2.2] as we are dealing with parallel weight Hilbert modular forms.

Let $\Lambda$ be the completed local ring of $\mathcal{W}$ at $\kappa(x)$ and let $m_{\Lambda}$ be its maximal ideal. It is isomorphic to a power series ring in $n+1$ variables over $\overline{\mathbb{Q}}_{p}$. The weight map $\kappa$ induces a finite homomorphism $\Lambda \rightarrow \mathcal{T}$ of local reduced complete rings. The algebra of the fiber of $\kappa$ at $x$ is given by $\mathcal{T}^{\prime}:=\mathcal{T} / m_{\Lambda} \mathcal{T}$ and it is a local Artinian $\overline{\mathbb{Q}}_{p}$-algebra. Observe that, the kernel of the surjective map $\mathcal{T} \rightarrow \mathcal{T}_{0}$ is contained in $m_{\Lambda} \mathcal{T}$ and hence, the surjective map $\mathcal{T} \rightarrow \mathcal{T}^{\prime}$ factors through $\mathcal{T}_{0}$. Thus, using the arguments of [4, Section 6] along with Proposition 4, we see that the representation $\rho_{\mathcal{T}^{\prime}}: G_{F} \rightarrow G L_{2}\left(\mathcal{T}^{\prime}\right)$, obtained by composing $\rho_{\mathcal{T}}$ with the surjective map $\mathcal{T} \rightarrow \mathcal{T}^{\prime}$, is an ordinary deformation of $\rho$ with constant determinant i.e. $\rho_{\mathcal{T}^{\prime}}$ is ordinary at every prime $\mathfrak{p}_{i} \mid p$, its reduction modulo the maximal ideal is $\rho$ and $\operatorname{det}\left(\rho_{\mathcal{T}^{\prime}}\right)=\operatorname{det}(\rho)$.

Let $\mathcal{D}, \mathcal{D}_{0}$ and $\mathcal{D}^{\prime}$ be the deformation functors described in the introduction ( $§ 1$ ) associated to $\rho$ and the unramified characters $\psi_{i}^{\prime \prime}$ such that $\psi_{i}^{\prime \prime}\left(\right.$ Frob $\left._{\mathfrak{p}_{i}}\right)$ is the $U\left(\mathfrak{p}_{i}\right)$-eigenvalue of $f_{\left(\gamma_{i}\right)}$ for $i=1, \cdots, r$ i.e. $\psi_{i}^{\prime \prime}\left(\right.$ Frob $\left._{\mathfrak{p}_{i}}\right)=\gamma_{i}$ for all $i$. As in the introduction ( $\S 1$, let $\mathcal{R}, \mathcal{R}_{0}, \mathcal{R}^{\prime}$ be the universal deformation rings representing the functors $\mathcal{D}, \mathcal{D}_{0}$ and $\mathcal{D}^{\prime}$, respectively. From Proposition 1 along with the discussion in the previous paragraph, we get continuous morphisms of local complete noetherian $\overline{\mathbb{Q}_{p}}$-algebras $\mathcal{R} \rightarrow \mathcal{T}, \mathcal{R}_{0} \rightarrow \mathcal{T}_{0}$ and $\mathcal{R}^{\prime} \rightarrow \mathcal{T}^{\prime}$.

Proposition 5. The morphisms $\mathcal{R} \rightarrow \mathcal{T}, \mathcal{R}_{0} \rightarrow \mathcal{T}_{0}$ and $\mathcal{R}^{\prime} \rightarrow \mathcal{T}^{\prime}$ are surjective. 
Proof. Same as that of Proposition 6.1 of [4] as we have established Proposition 4 above which is an analogue of [4, Proposition 5.1]. To be precise, we know that $\operatorname{Tr}\left(\rho_{\mathcal{T}}\right)\left(\right.$ Frob $\left._{\mathfrak{q}}\right)=T(\mathfrak{q})$ and for every $1 \leq i \leq r$, there exists a $y_{i} \in F_{\mathfrak{p}_{i}}{ }^{\times}$such that $\left(\psi_{\mathcal{T}}^{\prime \prime}\right)_{i} \circ \operatorname{Art}_{\mathfrak{p}_{i}}\left(y_{i}\right)=U\left(\mathfrak{p}_{i}\right)$, where $\operatorname{Art}_{\mathfrak{p}_{i}}: F_{\mathfrak{p}_{i}}{ }^{\times} \rightarrow \operatorname{Gal}\left(F_{\mathfrak{p}_{i}}^{a b} / F_{\mathfrak{p}_{i}}\right)$ is the local Artin map $\left(F_{\mathfrak{p}_{i}}^{\mathrm{ab}}\right.$ is the maximal abelian extension of $\left.F_{\mathfrak{p}_{i}}\right)$. This means that the image of the map $\mathcal{R} \rightarrow \mathcal{T}$ contains $T(\mathfrak{q})$ for primes $\mathfrak{q} \nmid N p$ and $U\left(\mathfrak{p}_{i}\right)$ for $1 \leq i \leq r$, which together generate $\mathcal{T}$. Hence, the map $\mathcal{R} \rightarrow \mathcal{T}$ is surjective. The surjectivity for the other two maps follows similarly.

Recall that $f$ and hence, $x$ is a point with parallel weights. From [14, Proposition 2.3], it follows that the largest quotient $\mathcal{T}^{\text {ord }}$ of $\mathcal{T}$ such that the composition of $\rho_{\mathcal{T}}$ with the quotient map $\mathcal{T} \rightarrow \mathcal{T}^{\text {ord }}$ is an ordinary deformation of $\rho$ should have "parallel weights" which means, following the notations of [1], the weights $\nu$ should be 0 and hence, the quotient map $\mathcal{T} \rightarrow \mathcal{T}^{\text {ord }}$ factors through $\mathcal{T}_{0}$. From Proposition 4 , it follows that the quotient map $\mathcal{T} \rightarrow \mathcal{T}_{0}$ factors through $\mathcal{T}^{\text {ord }}$. Hence, we get $\mathcal{T}^{\text {ord }}=\mathcal{T}_{0}$. From the arguments of [4, Section 6], we see that the largest quotient of $\mathcal{T}_{0}$ such that the composition of $\rho_{\mathcal{T}_{0}}$ with the quotient map is a deformation of $\rho$ with constant determinant is $\mathcal{T}^{\prime}$. Hence, the largest quotient of $\mathcal{T}$ such that the composition of $\rho_{\mathcal{T}}$ with the quotient map is an ordinary deformation of $\rho$ with constant determinant is $\mathcal{T}^{\prime}$.

\section{Proofs of Theorems 2, 3, 4}

We retain the notation of the previous sections here. So in particular, let $f$ be a classical, regular Hilbert modular form of parallel weight 1 defined over $F$ satisfying the conditions of one of the Theorems 2, 3, 4 and $\rho$ be the Galois representation attached to it. Let $f_{\left(\gamma_{i}\right)}$ be a $p$-stabilization of $f$ and let $x$ be the corresponding point of $\mathcal{E}$ as in the previous section. Let $\mathcal{T}, \mathcal{T}_{0}$ and $\mathcal{T}^{\prime}$ be the rings associated to $x$ that we introduced in the previous section.

Recall that each case of Theorem 1 corresponds to one of the Theorems 2, 3 and 4. The transcendence conjecture assumed in each case of Theorem 1 is also retained in its corresponding theorem. Hence, by Theorem 1 , the tangent space dimension of the universal nearly ordinary deformation ring $\mathcal{R}$ of $\rho$ occurring in Theorems 2 , 3, 4 is $n+1$, under the corresponding assumptions. By Proposition 5, we have a surjective map $R \rightarrow \mathcal{T}$. As $\mathcal{T}$ has Krull dimension $n+1$, it follows that the morphism $R \rightarrow \mathcal{T}$ is an isomorphism of regular local rings of Krull dimension $n+1$. Therefore, this proves the part of Theorems 2, 3, 4 regarding the smoothness of $\mathcal{E}$ at $x$. 
As we have established $\mathcal{R} \simeq \mathcal{T}$ in the cases considered in Theorems 2, 3, 4, it follows, from the last paragraph of the last section, that $\mathcal{R}_{0} \simeq \mathcal{T}_{0}$ and $\mathcal{R}^{\prime} \simeq \mathcal{T}^{\prime}$ in those cases under the corresponding assumptions. Recall that the Krull dimension of $\mathcal{T}^{\prime}$ and $\mathcal{T}_{0}$ is 0 and 1 , respectively. Therefore, combining the discussion above, we see that $\mathcal{C}^{\text {cusp }}$ is smooth at $x$ if and only if the dimension of the tangent space of $\mathcal{R}_{0}$ is 1 and the weight map $\kappa$ is étale at $x$ if and only if the tangent space dimension of $\mathcal{R}^{\prime}$ is 0 . We have calculated the dimensions of the tangent spaces of $\mathcal{R}_{0}$ and $\mathcal{R}^{\prime}$ in Theorem 1 in those cases under the same assumptions. Hence, we can conclude the parts of Theorems 2, 3, 4 regarding the étaleness of $\kappa$ and the smoothness of $\mathcal{C}^{\text {cusp }}$ at $x$ from Theorem 1 . This concludes the proofs of Theorems 2, 3, 4 .

\section{REFERENCES}

[1] F. Andreatta, A. Iovita, V. Pilloni, p-Adic families of Hilbert modular cuspform, To appear in Astérisque.

[2] B. Balasubramanyam, E. Ghate, V. Vatsal, On local Galois representations associated to ordinary Hilbert modular forms, Manuscripta Math. 142 (2013), no. 3-4, 513-524.

[3] J. Bellaïche and G. Chenevier, Families of Galois representations and Selmer groups, Astérisque 324, Soc. Math. France (2009).

[4] J. Bellaïche and M. Dimitrov, On the eigencurve at classical weight one point, Duke Math. J., 165. 2 (2016), 245-266.

[5] A. Betina, Les variétés de Hecke-Hilbert aux points classiques de poids 1, arxiv:1606.08901v1

[6] A. Brumer, On the units of algebraic number fields, Mathematika 14 (1967), 121-124.

[7] K. Buzzard, Eigenvarieties, Proceedings of the LMS Durham conference on L-functions and arithmetic geometry, 59-120, London Math. Soc. Lecture Note Ser. 320 (2007), Cambridge Univ. Press, Cambridge, 2007.

[8] F. Calegari and B. Mazur, Nearly Ordinary Galois Deformations over Arbitrary Number Fields, J. Inst. Math. Jussieu (2009) 8(1), 99-177.

[9] H. Carayol, Sur les représentations $\ell$-adiques associées aux formes modulaires de Hilbert, Ann. Sci. Éc. Norm. Supér. (4) 19 (1986), 409-468.

[10] S. Cho and V. Vatsal, Deformations of induced Galois representations, J. Reine Angew. Math., 556 (2003), 79-98.

[11] H. Darmon, A. Lauder and V. Rotger, Overconvergent generalised eigenforms of weight one and class fields of real quadratic fields, Advances in Mathematics 283 (2015), 130-142.

[12] M. Emsalem, H. Kisilevsky, D. Wales, Indépendance linéaire sur $\overline{\mathbb{Q}}$ de logarithmes p-adiques de nombres algébriques et rang p-adique du groupe des unités d'un corps de nombres, J. Number Theory 19 (1984), 384-391.

[13] H. Hida, On p-adic Hecke algebras for $G L_{2}$ over totally real fields, Ann. of Math. (2) 128 (1988), no. 2, 295-384.

[14] H. Hida, Nearly ordinary Hecke algebra and Galois representations of several variables, Algebraic analysis, geometry, and number theory (Baltimore, MD, 1988), 115-134, Johns Hopkins Univ. Press, Baltimore, MD, 1989.

[15] F. Jarvis, On Galois representations associated to Hilbert modular forms, J. Reine Angew. Math. 491 (1997), 199-216.

[16] M. Kisin and K.-F. Lai, Overconvergent Hilbert modular forms, Amer. J. Math. 127 (2005), no. $4,735-783$.

[17] D. Nelson, A variation on Leopoldt's conjecture: Some local units instead of all local units, arXiv: 1308.4637

[18] L. Nyssen, Pseudo-représentations, Math. Ann. 306 (1996), 257-283.

[19] M. Ohta, Hilbert modular forms of weight one and Galois representations, Progr. Math., 46 (1984), 333-353. 
[20] J. Rogawski and J. Tunnell, On Artin L-functions associated to Hilbert modular forms of weight one, Invent. Math. 74 (1983), no. 1, 1-42.

[21] R. Rouquier, Caractérisation des caractéres et pseudo-caractéres, J. Algebra 180(2) (1996), 571-586.

[22] R. Taylor, On Galois representations associated to Hilbert modular forms, Invent. Math, 98 (1989), 265-280.

[23] A. Wiles, On ordinary $\lambda$-adic representations associated to modular forms, Invent. Math. 94 (1988), 529-573.

E-mail address: deoshaunak@gmail.com

Université du Luxembourg, Faculté des Sciences, de la Technologie et de la Communication, 6, rue Richard Coudenhove-Kalergi, L-1359 Luxembourg, LuxemBOURG 